\title{
Preliminary Report upon the Fauna and Flora of Plymouth Sound.
}

By

\section{Walter Heape, M.A.}

THIs Preliminary Report upon the Fauna and Flora of Plymouth Sound is almost entirely composed of lists of species which have been recorded as either actually taken in Plymouth Sound, or common to the neighbourhood.

The list of Marine Algæ is a reprint of the late Mr. T. Boswarva's catalogue, with a few additions kindly furnished me by Mr. Holmes.

Mr. C. Spence Bate has been kind enough to prepare a list of Crustacea, and is largely responsible for the information upon that group of animals contained herein.

Mr. Baker has been good enough to provide a list of the Mollusca of the Sound and neighbouring coast, which has been of great use to me; while to several fishermen I am indebted for the names of some of the rarer fishes caught in the locality.

To all these gentlemen I would now express my best thanks.

\section{A CATALOGUE OF THE MARINE ALGA OF PLYMOUTH.}

By Mr. J. Boswarva.

Sub-Class I.-Melanospermea or Fucales.

(Olive Seaweeds.)

Order I. Fucaceæ.

Halidrys siliquosa (Podded Halidrys). In rock-pools, and on rocks, from Mount Batten outwards, at and below half-tide level. Perennial. Winter and spring. Cystoseira ericcides (Heath-like Cystoseira). From Bovisand outwards. Perennial.

Summer.

C. granulata (Granulated Cystoseira). From Bovisand outwards. Perennial. Summer.

VOL, I, NO. II. 
C. freniculacea (Fennel-leaved Cystoseira). From Bovisand outwards. Perennial. Summer.

C. fibrosa (Fibrous Cystoseira). From Bovisand outwards. Perennial. Summer.

Pycnophycus tuberculatus (Tubercled Pycnophycus). In rock-pools, from Mount Batten outwards. Perennial. Summer and autumn.

Fucus canaliculatus (Channelled Fucus). On rocks, between high water and halftide. Perennial. Summer and autumn.

$F$. nodosus (Knobbed Fucus). On rocks and large boulder stones. Perennial. Spring and summer.

F. serratus (Serrated Fucus). On rocks, half-tide level. Perennial. Winter and spring.

F. vesiculosus (Twin-bladdered Fucus). On rocks, stones, quays, \&c., exposed at low water. Perennial. Summer and winter.

F. ceranoides (Horn-like Fucus). In a brackish stream, Laira embankment. Perennial. Spring and summer.

Himanthalia lorea (Leather-thong Himanthalia). Abundant on the shore. Spring and summer.

\section{Order II. Sporochnaceæ.}

Desmarestia aculeata (Prickly Desmarestia). On rocks on the coast, near lowwater mark, and at a greater depth. Perennial.

D. ligulata (Tapering Desmarestia). On rocks on the coast, near low-water mark, and at a greater depth. Perennial.

D. viridis (Green Desmarestia). Firestone Bay; Mount Edgcumbe; low water. Annual. Spring and early summer.

Arthrocladia villosa (Hairy Arthrocladia). Sound. Annual. Summer.

Sporochnus pedunculatus (Pedunculated Sporochnus). Sound. Annual. Summer. Carpomitra Cabrera (Cabrera's Carpomitra). Sound. Annual. Summer.

\section{Order III. Laminariaceæ.}

Laminaria digitata (Fingered Laminaria). Common on rocks in deep water, and in pools. Perennial.

L. saccharina (Sugared Laminaria). Common at low-water mark, and in deep water. Perennial.

L. phyllitis. On rocks. Spring. Rare.

L. fascia (Band Laminaria). Mount Edgcumbe; Hoe; Mount Batton; and on buoys in the harbour.

Chorda filum (Thread Chorda). On rocks, stones, and in deep water. Annual. Summer and winter.

C. lomentaria (Jointed Chorda). On stones and rocks, Breakwater; Firestone Bay. Annual. Summer and autumn.

\section{Order IV. Dictyotaceæ.}

Cutleria multifida (Many-slit Cutleria). Dredged in the Sound, and washed on the shore. Perennial. Summer and autumn.

Haliseris polypodioides (Polypody-like Haliseris). Dredged in the Sound, and washed on the shore. Perennial. Summer and autumn.

Dictyota dichotoma (Forked Dictyota). In pools, and on rocks and stones. Annual. Summer.

Taonia atomaria (Banded Taonia). In tide-pools, and on rocks at low water. Bovisand and Whitsand Bay. Annual. Summer.

Stilophora rhizodes (Root-like Stilophora). Parasitical on algæ. Wembury Bay. Annual. Summer.

Dictyosiphon fæeniculaceus (Fennel Dictyosiphon). In pools, and on rocks and stones. Torpoint. Annual. Spring and summer.

Striaria attenuata (Tapering Striaria). In pools. Firestone Bay; Trevol; Hoe. Annual. Summer. 
Punctaria latifolia (Broad-leaf Punctaria). In pools, and on rocks. Mount Batten. Annual. Summer.

P. plantaginea (Plantain Punctaria). In pools, and on rocks. Mount Batten. Annual. Summer.

Asperococcus compressus (Compressed Asperococcus). In pools, and on rocks. Bovisand. Annual. Summer.

A. echinatus (Prickly Asperococcus). In pools, and on rocks. Firestone Bay; Hoe. Annual. Summer.

Litosiphon pusillus (Small Litosiphon). Parasitical on Himanthalia lorea. Annual. Summer.

\section{Order V. Chordariaceæ.}

Chordaria flagelliformis (Whip Chordaria). Cawsand; Bovisand. Annual. Summer. Mesogloia vermicularis (Worm-like Mesogloia). Common on the coast. Annual. Summer.

$M$. virescens (Pale green Mesogloia). Common on the coast. Annual. Summer.

M. Griffithsiana (Mrs. Griffith's Mesogloia). In rock-pools. Annual. Summer. Rare.

Leathesia tuberiformis (Tuber-shaped Leathesia). Wembury Bay; Mewstone. Annual. Summer.

L. Berkeleyi (Berkeley's Leathesia). Wembury Bay; Mewstone. Annual. Summer.

Elachista fucicola (Fucus inhabiting Elachista). Parasitical on Fuci. Annual. Summer.

E. flaccida (Flaccid Elachista). Parasitical on Cystoseira fibrosa. Annual. Summer.

E. scutulata (Little Shield Elachista). Parasitical on Dictyota dichotoma. Annual. Summer.

Myrionema strangulans (Choking Myrionema). Parasitical on Ulva and Enteromorpha.

\section{Order VI. Ectocarpaceæ.}

Cladostephus verticillatus (Whorled Cladostephus). Common. Whitsand Bay ; Mount Edgcumbe; Bovisand. Annual. Summer.

C. spongiosus (Spongy Cladostephus). Common. Hoe; Firestone Bay; Mount Edgcumbe; Bovisand. Annual. Summer.

Sphacelaria filicina (Fern-like Sphacelaria). Whitsand Bay; Mewstone; Sound. Annual. Summer.

S. scoparia (Broom Sphacelaria). Common on flat rocks. Annual. Summer.

S. plumosa (Feathery Sphacelaria). Mount Batten. Annual. Summer.

$S$. cirrhosa (Hair-like Sphacelaria). Rock-pools; parasitical on other algæ and corallines. Annual. Summer.

Ectocarpus siliculosus (Pod-fruited Ectocarpus). Parasitical on other algæ; sides of rocks. Summer.

E. fasciculatus (Fasciculate Ectocarpus). Parasitical on Laminaria. Summer.

E. Hincksia (Miss Hincks' Ectocarpus). Parasitical on Laminaria, in rock-pools. summer and autumn.

E. littoralis (Littoral Ectocarpus). Parasitical on algæ and Zostera, in rock-pools. Summer and autumn.

E. tessalatus (Tesselated Ectocarpus). Parasitical on algæ. Summer. Rare.

$E$. fenestratus (Windowed Ectocarpus). On rocks. Hoe. Annual. Summer.

E. tomentosus (Woolly Ectocarpus). Parasitical on Fucus vesiculosus. Common. Annual. Summer.

E. crinitus (Hairy Ectocarpus). On muddy sea-shores. Annual. Summer.

E. pusillus (Small Ectocarpus). Parasitical on small algæ. Firestone Bay. Annual. Summer.

E. granulosus (Granulous Ectocarpus). Parasitical on the smaller algæ. Common. Summer. 
E. spherophorus (Warted Ectocarpus). Parasitical on Fuci, at half-tide mark. Mewstone; Bovisand. Annual. Summer. Rare.

E. brachiatus (Cross-branched Ectocarpus). Parasitical on Rhodymenia palmata. Firestone Bay. Annual. Summer.

E. Mertensii (Mertens' Ectocarpus). In pools and on sand-covered rocks. Mount Edgcumbe; Whitsand Bay. A pril and May.

E. longifructus (Long-fruited Ectocarpus. On rocks. Hoe. Annual, Summer. Rare.

Myriotrichia clavaformis (Club-shaped Myriotrichia). Parasitical on Chorda lomentaria. Annual. Summer.

M. filiformis (Thread-like Myriotrichia). Parasitical on Chorda lomentaria. Annual. Summer.

\section{Sub-Class II.-Rhodospermez or Ceramiales. (Red or Brown Seaweeds.)}

\section{Order VII. Rhodomelaceæ.}

Rhodomela subfusca (Brownish Rhodomela). Common on rocks and shells between tide-marks. Perennial. Spring and summer.

Bostrychia scorpioides (Scorpioid Bostrychia). Hollows of sea-walls. Trevol; Pomfleet.

Rytiphløa pinustroides (Pine Rytiphlæa). On rocks, low-water mark. Whitsand Bay. Perennial. Autumn and winter.

R. thuyoides (Cypress Rytiphlæa). In tide-pools, and on corallines and flat rocks. Annual. Summer.

R. fruticulosa (Shrubby Rytiphlæa). In tide-pools, and on corallines. Mount Batten; Bovisand. Perennial. Summer.

R. complanata (Compressed or flattish Rytiphlæa). On rocks at low-water mark. Bovisand.

Polysiphonia urceolata (Pitchered Polysiphonia). Very common on rocks, stones, and in pools. Annual. Spring and summer.

$P$. formosa (Beautiful Polysiphonia). In tide-pools, on stones. Torpoint. Annual. Summer.

P. pulminata (Cushioned Polysiphonia). On rocks between tide-marks. Hoe; Whitsand Bay. Annual, Summer.

P. flbrata (Fibred Polysiphonia). On rocks, and in tide-pools. Mount Batten. Annual. Summer and autumn.

P. elongella (Divaricate Polysiphonia). On rocks, stones, and small algæ. Mount Edgcumbe. Biennial. Spring and summer.

$P$. elongata (Lobster-horn Polysiphonia). On rocks and shells, in tide-pools and deep water. Mount Batten; Mount Edgcumbe; Torpoint. Perennial. Spring and summer.

P. violacea (Violet Polysiphonia). On rocks and stones, low-water mark. Mount Edgcumbe; Bovisand. Annual. May and June.

$P$. fibrillosa (Fibrillose Polysiphonia). On rocks and stones, low-water mark. Mount Batten; Whitsand Bay. Annual. Summer.

P. Brodici (Brodie's Polysiphonia). On rocks, and in pools. Corbeal; Torpoint.

$P$. variegata (Variegated Polysiphonia). On mud-covered rocks. Beggar's Island; Torpoint.

$P$. simulans (Deceptive Polysiphonia). On rocks, and in tide-pools, low-water mark. Bovisand.

P. nigrescens (Blackish Polysiphonia). Common at half-tide mark. Perennial. Spring and summer.

P. atro-rubescens (Dark-red Polysiphonia). In pools, and on stones. Common. Annual. Spring and summer.

P. fastigiata (Level-topped Polysiphonia). On Fuci. Perennial. Summer and winter. 
P. fatidissima (Fetid Polysiphonia). Mount Edycumbe. Annual. Summer.

$P$. parasitica (Parasitic Polysiphonia). On corallines, in pools and deep water. Firestone Bay. Annual. Summer.

$P$. byssoides (Byssoid Polysiphonia). On rocks and stones, near low-water mark, and in deep water. Annual. Summer.

Dasya coccinea (Scarlet Dasya). Deep water. Annual. Summer and autumn.

D. ocellata (Ocellated Dasya). On mud-covered rocks. Beggar's Island; Torpoint. Annual. Summer.

D. arbuscula (Shrub Dasya). On rocks, low-water mark. Mewstone; Mount Edgcumbe. Annual. Summer.

\section{Order VIII. Laurenciacea.}

Bonnemaisonia asparagoides (Asparagus-like Bonnemaisonia). Sound, and washed on shore. Annual. June to September.

Laurencia pinnatifida (Pinnatifid Laurencia). Abundant on rocks.

L. caspitosa (Tufted Laurencia). On stones and rocks. Wembury Bay. Annual. Summer.

L. dasyphylla-[Chondria dasyphylla]-(Thick-leaved Laurencia). On rocks, lowwater mark. Bovisand; Mount Edgcumbe. Annual. Summer.

L. obtusa (Obtuse Laurencia). On the larger algæ. Annual. Summer and autumn.

L. tenuissima - [Chondria tenuissima]-(Slender Laurencia). On rocks, low-water mark. Bovisand. Annual. Summer.

Chrysymenia clavellosa-[Chylocladia clavellosa]-(Clubbed Chrysymenia). Abundant in pools and deep water. Annual. May to September.

C. rosea-[Chylocladia rosea]-(Rosy Chrysymenia). In pools, and on sides of rocks. Mount Edgcumbe; Firestone Bay. January to June. Rare. Taken by J. Gatcombe.

Chylocladia ovalis - [Lomentaria ovalis $]-($ Oval-leaved Chylocladia). On rocks, deep water, and on other algæ. Mount Edgcumbe; Firestone Bay. Annual. Spring.

C. kaliformis - [Lomentaria kaliformis]-(Whorled Chylocladia). On rocks, deep water, and on other algæ. Mount Edgcumbe; Firestone Bay. Annual. Summer.

C. reflexa-[Lomentaria reflexa]-(Reflexed Chylocladia). Occasionally in tidepools. Annual. Summer. Rare.

C. parvula-[Champia parvula]-(Small Chylocladia). On small algæ and corallines. Firestone Bay. Annual. Summer and autumn.

C. articulata (Jointed Chylocladia). On rocks and algæ. Annual. Spring and summer. Common.

\section{Order IX. Corallinaceæ.}

Corallina officinalis (Medicinal Coralline). On rocks, and in pools between tidemarks. Common. Perennial.

C. squamata (Scaled Coralline). On rocks, and in pools between tide-marks. Common. Perennial.

Jania rubens (Red Jania). On small algæ. Bovisand and Whitsand Bay. Annual. Summer.

Melobesia calcarea (Chalk Melobesia). On rocks round the coast. Perennial.

$M$. membranacea (Membranaceous Melobesia). On rocks round the coast. Perennial.

M. polymorpha (Many-shaped Melobesia). On rocks round the coast. Perennial.

M. pustulata (Pimpled Melobesia). On Phylophora rubens and other algæ. Annual. Summer.

Hildenbrandtia rubra (Red Hildenbrandtia). On smooth stones, pebbles, and deep water. At all seasons. 


\section{Order X. Delesseriaceæ.}

Delesseria sanguinea-[Wormskioldia sanguinea]-(Blood-red Delesseria). In pools, sea, and on the sides of rocks. Abundant. Perennial. Spring and summer; fruiting in winter on old stems.

D. sinuosa (Sinuous Delesseria). On larger algæ, in deep water. Biennial. Autumn and winter.

D. alata (Winged Delesseria). On rocks. Bovisand; Redding Point. Biennial. Summer.

D. hypoglossum (Proliferous Delesseria). On rocks and algæ, and in deep water. Annual. Spring and summer.

D. ruscifolia (Obtuse-leaved Delesseria). Sides of rocks, and on stones. Annual. Summer and autumn.

Nitophyllum punctatum (Dotted Nitophyllum). In deep water. Mount Edgeumbe ; Firestone Bay. Annual. Summer.

N. Hillia (Miss Hill's Nitophyllum). In deep water. Mount Edgcumbe; Mount Batten. Annual. Summer.

N. Bonnemaisoni (Bonnemaison's Nitophyllum). Mount Edgcumbe, and on the stems of Laminaria. Annual. Summer.

N. Gmelini (Gmelin's Nitophyllum). Mount Edgcumbe ; Anthony-passage ; Firestone Bay. Annual. Summer.

N. laceratum (Torn Nitophyllum). Sides of rocks, deep water. Common. Annual. Summer.

$N$. versicolor (Changeable Nitophyllum). Bovisand, and deep water. Annual. Summer.

Plocamium coccineum (Scarlet Plocamium). On rocks and algæ. Common. Perennial. Summer.

\section{Order XI. Rhodymeniaceæ.}

Stenogramme interrupta (Interrupted Stenogramme). Dredged in summer, and washed on shore at different seasons of the year. Sound. Perennial. Rare.* Taken by J. Gatcombe.

* “This very interesting plant, by far the most important addition lately made to the British Marine Flora, was discovered on the 21st October, 1846, by Dr. John Cocks, of Plymouth, among rejectamenta on the shore at Bovisand, near Plymouth. A few days subsequently it was met with in a neighbouring station by the Rev. W.S. Hore, who at the same time gathered the equally rare and curious Carpomitra Cabrerce; and to the untiring perseverance of both these gentlemen, who, day by day, during the inclement month of November-in all weathers-visited the shore, and preserved every scrap of these plants which the waves threw up, we are indebted for all the British specimens which have yet been taken of the Stenogramme, and for all, except Miss Ball's original one, of the Carpomitre."-Harvey's Phycologia Britannica, 1851.

Rhodymenia bifida-[Rhodophyllis bifida]-(Cloven Rhodymenia). On sides of rocks and stones, in deep water. Annual. Summer and autumn.

R. bifida var. cristata- [Euthora cristata]-On sides of rocks and stones, in deep water. Annual. Summer and autumn.

R. laciniata [Callophyllis laciniata]-(Jagged Rhodymenia). On rocks and stones in the sea, and on Laminaria. Spring and summer.

R. ciliata [Calliblepharis ciliata]-(Ciliated Rhodymenia). On rocks at low-water mark, and in deep water. Annual. Summer and winter.

R. palmetta (Little-palm Rhodymenia). On rocks at low-water mark, also in pools, and on stems of Laminaria. Annual. Summer and autumn.

R. palmata (Dulse or Dillisk Rhodymenia). On rocks and Laminaria. Abundant. Perennial.

R. jubata-[Calliblepharis jubata]-(Cirrhose Rhodymenia). In pools, helow halftide. General. Annual. Summer. 
Sphærococcus coronopifolius (Buck's-horn Sphærococcus). Sound. Summer and winter.

Gracilaria confervoides (Conferva-like Gracilaria). On rocks and stones on the shore. General. Spring to winter.

G. multipartita (Many-divided Gracilaria). On rocks and stones on the shore. Tait's Hill; Firestone Bay. Perennial. Summer and winter.

G. erecta-[Cordylecladia erecta]-(Erect Gracilaria). Torpoint. Perennial. Fruiting in winter.

Cystoclonium purpurascens - [old name, Hypnea purpurascens]-(Purple Cystoclonium). Mount Batten; Redding Point. Annual. Summer.

\section{Order XII. Cryptonemiaceæ.}

Grateloupia filicina (Fern-like Grateloupia). Whitsand Bay; Mount Batten Perennial. Winter and spring.

Gelidium corneum (Horny Gelidium). On rocks and in pools. General. Annual. Summer. Several varieties.

Gigartina pistillata (Pedicellate Gigartina). Whitsand Bay. Perennial. Summer and autumn. Rare.

G. acicularis (Needle-branched Gigartina). Bovisand; Redding Point. Annual. Winter. Rare.

G. mamillosa (Mamillose Gigartina). General, On rocks. Perennial. Winter and summer. Carrigeen Moss.

Chondrus crispus (Curled Chondrus). General. On rocks and in pools. Perennial. Spring and summer. Carrigeen Moss.

C. norvegicus - [Gymnogongrus norvegicus]-(Norwegian Chondrus). In pools ; occasionally on shore-rocks. Perennial. Autumn to summer.

Phyllophora rubens (Red Phyllophora). Whitsand Bay. Perennial. Winter and spring.

P. membranifolia (Membrane-leaved Phyllophora). In rock-pools, but not frequent.

P. Brodicei (Brodie's Phyllophora). On Laminaria. Whitsand Bay. Perennial. Winter and spring.

P. palmettoides (Palmetta-like Phyllophora). Whitsand Bay. Perennial. Winter and spring. Rare.

Gymnogongrus Griffithsia (Mrs. Griffiths's Gymnogongrus). Cawsand Bay ; Mount Edgcumbe. Perennial. Autumn and winter. Rare.

G. plicatus - [Ahnfeldtia plicata]-(Entangled Gymnogongrus). In shallow rockpools. Bovisand; Mount Edgcumbe. Perennial. Common.

Polyides rotundus (Round Polyides). In pools and running water. Common. Perennial. Winter and spring.

Furcellaria fastigiata (Pointed Furcellaria). Half-tide mark. Mount Batten. Perennial. Winter.

Dumontia filiformis (Thread-like Dumontia). General. Annual. Summer.

Halymenia ligulata (Strap-shaped Halymenia). Deep water. Annual. Summer.

Ginannia furcellatu-[Scinaia furcellata $]$-(Forked Ginannia). Deep water, and on rocks. Whitsand Bay. Annual. Summer.

Kallymenia reniformis (Kidney-shaped Kallymenia). Deep water, and at low-water mark. Mount Edgcumbe. Perennial. Summer and autumn.

K. Dubyi-[Schizymenia Dubyi]-(Duby's Kallymenia), Firestone Bay; Hoe ; Mount Edgcumbe; Mount Batten. Annual. Winter and spring.

Iridcea edulis - [Schizymenia edulis]-(Edible Iridæa). On the coast, and in deep water. General. Perennial. Autumn and winter.

Catenella Opuntia (Indian-fig Catenella). Faces of rocks. Hoe; Mount Edgcumbe. Perennial.

Naccaria Wiggii (Wigg's Naccaria). Sound. Annual. Summer. Rare.

Glorosiphonia capillaris (Slender Gloiosiphonia). In tide pools. Mount Edgcumbe; Wembury Bay. Annual. Early summer.

Nemaleon multifidum (Many-slit Nemaleon). On rocks, at low-water mark. Cawsand Bay; Bovisand. Summer. 
N. purpureum-[Helminthocladia purpurea $]$-(Purple Nemaleon). On rocks. Whitsand Bay. Annual. Summer.

Dudresnaia coccinea (Scarlet Dudresnaia). Sound. Annual. Summer. Rare.

D. divaricata- $[$ Helminthora divaricata $]$-Divaricate Dudresnaia). Wembury Bay. Annual. Summer and autumn.

Crouania attenuata (Attenuated Crouania). On corallines and small algæ. Firestone Bay. Annual. Autumn. Very rare.

\section{Order XIII. Ceramiaceæ.}

Ptilota sericea-[Ptilota elegans]-(Silken Ptilota). On rocks, and in pools. General. Perennial. Summer and autumn. An elegant variety is sometimes found on the outer shores, which approaches very near to Ptilota plumosa.

Microcladia glandulosa (Glandular Microcladia). On Rhodymenia laciniata and Ceramium rubrum. Sound. Annual. From March to September. Very rare. Taken by J. Gatcombe.

Ceramium rubrum (Red Ceramium). In pools and deep water on the shores. General. Perennial. All seasons.

C. decurrens (Decurrent Ceramium). Bovisand. Annual. Autumn. Rare.

C. Deslongchampsii (Deslongchamps' Ceramium). On stones, at half-tide mark. Mount Edgcumbe. Annual. Spring.

C. diaphanum (Diaphanous Ceramium). On rocks, and in tide-pools. Bovisand; Mewstone. Annual. Summer.

C. gracillimum (Very slender Ceramium). At low-water mark. Mount Batten ; Bovisand. Annual. September. Rare.

C. flabelligerum (Fan-bearing Ceramium). Parasitical on Cladostephus spongiosus. Hoe. Annual. Spring.

C. fastigiatum (Level-topped Ceramium). On rocks, at low-water mark. Mount Batten. Firestone Bay. Annual. Autumn and winter. Rare.

C. nodosum (Knobbed Ceramium). Washed up from deep water. Annual. Summer.

C. strictum (Straight Ceramium). On rocks, at half-tide. General. Annual. Spring and summer.

C. echionotum (Prickly Ceramium). Deep water. Bovisand; Mount Edgcumbe. Annual. Summer.

C. acanthonotum (One-spined Ceramium). On rocks. General. Annual, Spring and summer.

C. ciliatum (Ciliated Ceramium). In pools. General. Annual. Spring and summer.

Spyridia filamentosa (Filamentose Spyridia). At low-water mark. Firestone Bay ; Bovisand. Annual. Summer.

Griffithsia equisetifolia (Equisetum-leaved Griffithsia). Low-water mark. Bovisand; Whitsand Bay. Perennial. Summer.

G. devoniensis (Devonshire Griffithsia). Low-water mark. Mount Edgcumbe; Torpoint; Beggar's Island. Annual. Summer.

G. corallina (Coral-like Griffithsia). On rocks, near low-water mark. Annual. Summer.

G. secundiflora (Side-fruited Griffithsia). On one rock, at low-water mark. Bovisand. Annual. Spring and summer. Very rare. First found by Rev. W. S. Hore.

G. setacea (Bristly Griffithsia). On rocks, and in pools. General. Perennial. Fruiting in winter.

Wrangelia multifida (Many-slit Wrangelia). On rocks. Mount Edgcumbe; Mount Batten; Bovisand. Ánnual. Summer.

W. multifida, var, pilifera. On rocks. Mount Edgcumbe; Mount Batten; Bovisand. Annual. Summer.

Seirospora Griffithsiana (Mrs. Griffiths's Seirospora). Sound. Summer. Annual. Very rare. Taken by J. Gatcombe. 
Callithamnion Plumula (Little Feather Callithamnion). General. Annual. Spring to winter.

C. cruciatum (Crossed Callithamnion). Torpoint. Annual. Summer.

C. Turneri (Turner's Callithamnion). Bovisand. Annual. Summer.

C. barbatum (Bearded Callithamnion). Hoe. Annual. Summer.

C. pluma (Feather Callithamnion). On stems of Laminaria. Summer.

C. Brodici (Brodie's Callithamnion). Torpoint. Annual. Summer.

C. tetragonum (Four-angled Callithamnion). Bovisand. Annual. Summer.

C. brachiatum (Armed Callithamnion). On Laminaria. Wembury Bay.

C. tetricum (Rough Callithamnion). On sides of rocks. General. Annual. Summer,

C. Hookeri (Hooker's Callithamnion). On rocks. Hoe. Annual. Summer.

C. roseum (Rosy Callithamnion). On mud-banks and stones. Annual. Spring and summer.

C. byssoideum (Byssus-like Callithamnion). On corallines and stones. Annual. Spring.

C. polyspermum (Many-seeded Callithamnion). On Fuci. Mount Edgcumbe. Annual. Spring.

C. Borreri (Borrer's Callithamnion). On rocks and stones. Mount Batten; Torpoint; Anthony-passage. Annual. Summer. Rare.

C. gracillimum (Very graceful Callithamnion). Mount Batten. Annual. Autumn.

C. thuyoideum (Cypress Callithamnion). On sides of rocks. Hoe; Mount Edgcumbe. Annual. Summer.

C. corymbosum (Corymbose Callithamnion). Torpoint. Annual, Spring.

C. spongiosum - [Callithamnion granulatum]-(Spongy Callithamnion). Bovisand; Firestone Bay. Annual. July and Augnst.

C. pedicellatum - [Corynespora pedicellata $]$-(Pedicellate Callithamnion). In rockpools. Cremill ; Mount Batten. Annual. Summer.

C. Rothii (Roth's Callithamnion). Bovisand Pier. Perennial. Winter.

C. floridulum (Gay Callithamnion). In pools. Hoe. Annual. Summer.

C. Daviesii (Davies' Callithamnion). Parasitical on Ceramium rubrum. Annual. Summer.

C. virgatulum (Little twig Callithamnion). Parasitical on Ceramium rubrum. Annual. Summer.

\section{Sub-Class III.-Chlorosperma or Confervales. (Grass-green Seaweeds.)}

\section{Order XIV. Siphonaceæ.}

Codium Bursa (Purse Codium). One found on a buoy in Stonehouse Pool. Summer. C.adhcerens (Adhering Codium). Wembury. Annual. Summer.

C. tomentosum (Tomentose Codium). At low-water mark. Bovisand. Annuai. Summer.

Bryopsis plumosa (Feathery Bryopsis). General. Seldom appearing in the same place two following years. Annual. Summer.

B. hypnoides (Hypnum-like Bryopsis). Trevol; Mount Batten. Annual. Summer and autumn.

\section{Order XV. Confervaceæ.}

Cladophora pellucida (Transparent Cladophora). In rock-pools, and on stones. Firestone Bay; Hoe. Annual. Spring and summer.

C. Hutchinsiae (Miss Hutchins' Cladophora). In rock-pools, and on stones. Firestone Bay; Hoe. Annual. Spring and summer.

C. diffusa (Diffused Cladophora). In rock-pools, and on stones. Bovisand. Annual. Spring and summer.

C. gracilis (Slender Cladophora). In rock-pools, and on stones. Firestone Bay. Annual. Spring and summer. 
C. refracta (Reflexed Cladophora). In rock-pools and on stones. Whitsand Bay. Annual. Spring and summer.

C. albida (Whitish Cladophora). In rock-pools, and on stones. Bovisand; Cawsand Bay. Annual. Spring and summer.

C. lanosa (Woolly Cladophora). On other algæ. Bovisand; Stoke Bay. Annual. Spring and summer.

C. uncialis (Inch Cladophora). On rocks partly embedded in sand. Bovisand. Annual. Spring and summer.

C. arcta (Straight Cladophora). On rocks. Hoe; Bovisand. Annual. Spring and summer.

C. glaucescens (Glaucous Cladophora). On rocks. Firestone Bay; Hoe. Annual. Spring and summer.

C. rupestris (Rock-inhabiting Cladophora). On rocks, and in pools. Very general. Annual. Spring and summer.

C. leetevirens (Pale green Cladophora). On rocks. Hoe; Whitsand Bay. Annual. Spring and summer.

C. flexuosa (Flexuous Cladophora). On rocks. Mount Batten. Annual. Spring and summer.

Conferva tortuosa (Twisted Conferva). On rocks around the coast. Annual. Summer.

C. melagonium (Dark green Conferva). In rock-pools. Whitsand Bay. Annual. Summer.

C. area (Pale green Conferva). On sand-covered rocks. Whitsand Bay. Annual. Summer.

\section{Order XVI. Ulvaceæ.}

Enteromorpha intestinalis (Intestinal Enteromorpha). On rocks and stones, and in rock-pools on beaches. Annual. Summer.

E. compressa (Compressed Enteromorpha). On rocks and stones, and in rock-pools on beaches. Annual. Summer.

E. Linkiana (Link's Enteromorpha). On stones, in the sand. Mount Edgcumbe. Annual. Summer.

E. erecta (Erect Enteromorpha). On rocks in the sea. Annual. Summer.

Ulva latissima (Very broad Ulva). In rock-pools, and on rocks. Annual. Summer.

U. lactuca (Lettuce Ulva). On rocks. Cawsand Bay; Bovisand. Annual. Summer.

U. Linza (Narrow Ulva). In rock-pools, and on rocks. Annual. Summer.

Porphyra laciniata (Laciniated Porphyra). On stones and rocks, Annual. Summer.

P. vulgaris (Common Porphyra). On stones and rocks. Annual. Summer.

Bangia fusco-purpurea (Brown-purple Bangia). On rocks. Summer.

B. ciliaris (Fringe-like Bangia). On rocks. Mount Batten. Summer.

\section{Order XVII. Oscillatoriacex.}

Rivularia nitida (Glossy Rivularia). On rocks. Bovisand. Annual. Summer.

Calothrix confervicola (Conferva Calothrix). On Ceramiam rubrum. Mount Edg. cumbe. Annual. Summer.

Lyngbya majuscula (Large Lyngbya). Washed up from deep water. Mount Edgcumbe.

The following additional names of species observed by Mr. Holmes in Plymouth

Sound have been kindly forwarded to me by that gentleman:

Mitophyllum venulosum. Torpoint mud bank in June.

Arthrocladia villosa. Firestone Bay only.

Sporochines pedunculata. Torpoint.

Gratelonpia dichotoma. Renny Rocks.

Nannaria Wiggii. Torpoint. 
Ginnania funellata. Torpoint.

Unangelia multifida. Torpoint.

Callithannia venicolor. Torpoint.

And the following localities especially rich in seaweed mentioned by him :

Beggars Island and Rat Island, at the mouth of St. Germans River.

Treval mill pond, Hamoaze.

Renny Rocks, Wenbury Bay.

Mewstone.

Laira.

Mount Batten.

Mount Edgcumbe Beach.

Barn Pool.

Mill Bay.

The following species named in the foregoing list have other names.

RHODOSPERMEA.

Laurencia dasyphylla

L. tenuissima

$=$ Chondria dasyphylla.

Chrysymenia clavellosa

$=$ Chondria tenuissima.

C. rosea

$=$ Chylocladia clavellosa.

Chylocladia ovalis

C. kaliformis

C. reflexa

$=$ Chylocladia rosea.

$=$ Lomentaria ovalis.

C. parvula

= L. kaliformis.

$=$ L. reflexa.

Delesseria sanguinea

Rhodymenia cristata

R. bifida

R. laciniata

R. ciliata

R. jubata

Gracilaria erecta

Cystoclonium pupurascens

Chondrus norvegicus

Gymnogongrus plicatus

Ginannia furcellata

Kallymenia Dubyi

Iridæa edulis

Nemaleon purpureum

Dudresnaia divaricata

Ptilota sericea

Callithamnion spongiosum

C. pedicellatum

Cruoria pellita

$=$ L. parvula.

$=$ Wormskioldia sanguinea.

$=$ Euthora cristata.

$=$ Rhodophyllis bifida.

$=$ Callophyllis laciniata.

$=$ Calliblepharis ciliata.

$=$ C. jubata.

$=$ Cordylecladia erecta.

$=$ Hypnea purpurascens.

$=$ Gymnogongrus norvegicus.

$=$ Ahnfeldtia plicata.

$=$ Scinaia furcellata.

$=$ Schizymenia Dubyi.

$=$ S. edulis.

$=$ Helminthocladia purpurea.

$=$ Helminthora divaricata.

$=$ Ptilota elegans.

$=$ Callithamnion granulatum.

$=$ Corynespora pedicellata.

$=$ Petrocelis cruenta.

\section{PROTOZOA.}

Foraminifera.

Imperforata.

Biloculina (D'Orbig.).

ringens (D'Orbig.). In the Sound and at the Eddystone. var. carinata (D’Orbig.).

Miliolina (Will.). trigonula (Lam.).

Spirulina (Ehren.). foliacea (Phiilips). 
Lagena (Walk.).

\section{Perforata.}

vulgaris (Planci).

var. clavata (Willm.).

var. pellucida (Willm.).

var. striata (Willm.).

Entosolenia (Ehren.). globosa (Walk.), var. lineata (Willm.).

marginata (Walk.), var. lucida (Willm.).

squamosa (Maton and Rackett).

var. lagenoides (Willm.).

Lìngulina (D’Orbig.).

carinata (D'Orbig.).

Nodosaria (Lam.).

radicula (Linn.).

pyrula (D'Orbig.).

Dentalina (D'Orbig.).

subacuata (Mont.).

Cristellaria (Lam.). calcar (Linn.). subarcuatula (Walk.).

Polymorphina (D'Orbig.).

lactea (Adams).

var. oblonga (Brown). Near Eddystone.

var. communis (D'Orbig.).

myristiformis (Willm.). Near Eddystone.

Orbulina (D'Orbig.). universa (D'Orbig.).

Rotallina (D'Orbig.).

becarii (Linn.). Common on oyster-shells.

oblonga (Willm.). nitida (Willm.).

Planorbulina (D'Orbig.).

mediterranensis (D'Orbig.). Near Eddystone in abundance.

Globigerina (D'Orbig.). bulloides (D'Orbig.).

Truncatulina (D'Orbig.).

lobatula (Walker). Attached to Sertularians in coralline zone

Bulimina (D'Orbig.).

pupoides (D’Orbig.).

var. marginata (Willm.).

var. elongata (Willm.).

scabra (Willm.).

Textularia (Defr.).

cuneiformis (D'Orbig.).

variabilis (Willm.).

Nonionina (D'Orbig.).

crassula (Walk.). Common on oysters. jeffreysii (Willm.).

Polystomella (Lam.).

umbilicatula (Walk.) On oysters.

\section{PORIFERA.}

\section{Calcarea.}

Grantia (Flem.).

compressa (Flem.). Very common.

ciliata (Johns.). 
Leucosolenia (Bow.).

botryoides (Bow.).

Leuconia (Grant).

fistulosa (Bower.). Eddystone rocks.

Clathrina.

clathrus. Under sides of rocks. Hoe. Rare. (Stewart.)

Silicea.

Pachymatisma (Bow.).

johnstonia (Bow.). Wenbury Bay. (Stewart.)

Tethea (Lam.).

lyncurium (Johns.).

Hymeniacidon (Bow.).

suberea (Bow.). Common.

carnosua (Bow.).

celata (Bow.). Stone of Cremyll beach.

medius (Bow.).

Halichondria (Flem.). panicea (Johns.). Common.

Isodictya (Bow.). invalida (Bow.). lobata (Mont.).

Desmacidon (Bow.). fruticosa (Bow.).

\section{Keratosa.}

Chalina (Grant).

montaguii (Bow.).

\section{CELENTERATA.}

\section{Actinozoa.}

\section{Octactinia.}

Alcyonium.

digitatum (Linn.). Common in deep water off the coast.

Gorgonia (Edw.).

verrucosa (Linn.). Common in deep water off the coast.

\section{Hexactinia.}

Actinoloba (Blainv.).

dianthus (Blainv.). In the Sound.

Sagartia (Gosse).

bellis (Gosse). Deep pools, Whitsand Bay.

miniata (Gosse). In the Sound.

rosea (Gosse). Recorded from the neighbourhood by Rogers.

ichthystoma (Gosse). Deep water.

venusta (Gosse). In the neighbourhood (Rogers).

nivea (Gosse). Crevices and rock-pools in the Sound.

sphyrodeta (Gosse). Crevices and pools at low-water mark.

pallida (Gosse). In the neighbourhood (Rogers).

viduata (Gosse). Whitsand Bay.

parasitica (Gosse). Coralline zone.

troglodytes (Gosse). In the neighbourhood (Rogers).

Adamsia (Forb.)

palliata (Johnst.). Deep water. 
Anthea (Johnst.).

cereus (Johnst.). Common in Plymouth Sound.

Aiptasia (Gosse). couchii (Gosse). In the neighbourhood (Rogers).

Actinia (Linn.). mesembryanthemum (Ellis and Sol.). Common at low-water mark.

Bolocera (Gosse). tuediæ (Gosse). Trawled in deep water.

Bunodes (Gosse). gemmacea (Gosse). Common in deep pools. ballii (Gosse). In holes in rocks at low-water.

Tealia (Gosse). crassicornis (Gosse). Common in tide-pools.

Peachia (Gosse). hastata (Gosse). In the neighbourhood (Rogers).

Halcampa (Gosse). chrysanthellum (Gosse). In deep pools with sandy bottom. Whitsand Bay.

Edwardsia (Quatr.). carnea (Gosse). In the neighbourhood (Rogers).

Cerianthus (Della Chiaje). lloydii (Gosse). In the neighbourhood (Rogers).

Corynactis (Allm.). viridis (Allm.). Very common and in great variety.

Zoanthus (Cuv.). couchii (Johnston). In the neighbourhood (Rogers). rubicornis (Houldsw.). Within the Sound in twenty fathoms.

Caryophyllia (Lam.). smithii (Johns.). Fairly common at low-water mark on rocks in the Sound.

Balanophyllia (Wood.). regia (Gosse). One specimen has been found in the Sound.

Clava (Gmelin). multicornis (Pallas). Between tide-marks.

Hydractinia (v. Beneden). echinata (Flem.). On old shells.

Coryne (Gaert.). vaginata (Ehren.). In tide-pools.

Eudendrium (Ehren.). ramosum (Linn.). On Sertularians or alone. capillare (Alder). In Sound.

Tubularia (Linn.). indivisa (Linn.). Between tide-marks and in deep water. larynx (Ellis). In rock-pools and on Sertularians from deep water. gracilis (Harvey).

Corymorpha (Sars). nutans (Sars.). On sand, in six fathoms. Whitsand Bay.

Syncoryne (Ehren.). eximia (Allm.). Along the coast.

Bimeria (S. Wright). vestita (Wright). Along the coast.

Bougainvillia (Lesson). ramosa (v. Beneden). Along the coast.

Cladonema (Dujard.). radiatum (Dujard.). Along the coast. 
Myriothela (Sars.). phrygia (Fabric.). Along the coast.

Clavatella (Hincks). prolifera (Hincks). Along the coast.

Plumularia (Lam.).

\section{Calyptoblastea.} myriophyllum (Linn.). Common in trawl refuse. setacea (Ellis). echinulata (Lamk.). Abundant in tide-pools. similis (Hincks). Abundant on weed.

Sertularia (Linn.). polyzonias (Linn.). Between tide-marks and in deep water. gayi (Lamouroux). Trawl refuse from deep water. rosacea (Linn.). Near Plymouth. tamarisca (Linn.). Near Plymouth. abietina (Linn.). Common on the coast. argentea (Ellis and Sol.). Common on the coast. falcata (Linn.). Common.

Antennularia (Lam.). ramosa (Lam.). Near Plymouth. Not common.

Laomedea (Lam.). dichotoma (Linn.). On other zoophytes. longissima (Pallas van B.). In coralline zone. flexuosa (Hincks). Between tide-marks.

Campanularia (Lam.). volubilis (Linn.). In Sound in deep water. johnstonii (Alder). Between tide-marks and in deep water. hincksii (Alder). Between tide-marks and in deep water. verticillata (Linn.). In coralline zone.

Calycella (Hincks). dumosa (Flem.). Near Plymouth very fine. fruticosa (Sars.). In Sound or neighbourhood.

\section{ECHINODERMATA.}

Antedon (Frem.).

\section{Crinoidea.} rosacea (Linck.). Rocks of Drake's Island and off the Cobbler Buoy in the Sound (Stewart). Also rocks south-west of Eddystone in forty fathoms.

\section{Asteroidea.}

Asterias (Linn.). rubens (Linn.).

violacea (Müll.). Trawled in shallow water. glacialis (Linn.). In the neighbourhood (Rogers).

Asterina (Nardo). gibbosa (Penn.). Rock-pools.

Goniaster (Ag.). equestris (Gmel.). In the neighbourhood (Rogers).

Solaster (Forb.). papposus (Linn.).

Porania (Gray). pulvillus (Gray). In the Sound.

Astropecten (Linck.). irregularis (Penn.). Sandy shores. 
Cribrella (Ag.). oculata (Penn.). Plentiful.

Luidia (Forb.). savignii (And.). Taken off Plymouth. sarsii (Duben and Koren). Taken off Plymouth.

Ophiura (Lam.).

\section{Ophiuroidea.}

texturata (Lam.). Sandy shores.

albida (Forb.). In the neighbourhood (Rogers).

Ophiocoma (Ag.).

neglecta (Johnston). Under stones under the Hoe (Bellamy).

filiformis (Müll.). Inside east end of breakwater (Stewart). granulata (Link.). Off Plymouth in deep water (Bellamy).

Ophiothrix (M. Tr.).

fragilis (Müll.). Common.

Echinus (Bond).

\section{Echinoidea.} esculentus (Penn.). In the Sound. acutus (Lam.). In the neighbourhood (Rogers).

Echinocyamus (Leske). pusillus (Müll.). Dredged in crevices of stone.

Spatangus (Klein). purpureus (Müll.). Off Plymouth, occasional.

Amphidotus. cordatus (Penn.). Very common.

Holothuria (Linn.).

\section{Holothuroidea.} nigra (Peach). Not uncommon.

Cucumaria (Blainv.). pentactes (Müll.) Frequent. hyndmani (Thompson). In holes outside Breakwater.

Ocnus (Forb. and Good.). brunneus (Forbes). Dredged in Sound. Not uncommon.

Synapta (Esch.). digitata (Montg). On rocky shores.

Thyone (Oken).

papillosa (Müll.). In the neighbourhood (Rogers.).

\section{VERMES. \\ Platyhelminthes. \\ Turbellaria.}

Leptoplana (Hempr., Ehrenb.).

tremellaris (O. F. Müll.). Fairly numerous on seaweed, on rocks in Sound.

\section{Nemertina.}

Nemertes (Cuv.). borlasii (Cuv.). In the neighbourhood (Rogers). neesii (Oerst). ? Rocks. Drake's Island.

Micrura (Ehrb.). fasciolata (Ehrb.). Trawled in the Sound.

Amphiporus (Ehrb.). pulcher (O. F. Müll.). Rocks. Drake's Island. 
Sipunculus (Linn.).

\section{Gephyrea.}

nudus (Linn.). In holes in rocks inside Breakwater.

bernhardus. In holes in rocks inside Breakwater.

Thalassema (Gaertu).

neptuni (Gaertu.). In holes in rocks outside Breakwater.

\section{Annelida. \\ Hirudinea.}

Pontobdella (Leach).

areolata (Leach). Recorded from the Sound.

muricata (Lam.). In the neighbourhood (Rogers).

verrucata (Grube). On skate from deep water.

Tubifex (Lam.).

\section{Chætopoda.} lineatus (O. F. Müll.). Amongst fuci in coralline zone.

Terebella (Mont.). conchilega (Pall.). In the neighbourhood (Rogers).

Arenicola (Lam.). piscatorum (Lam.). Common.

Cirratulus (Lam.). cirratus (O. F. Müll.). Plentiful in mud under stones.

tentaculatus (Mont.). Plentiful in mud under stones, Jennycliffe Bay, the Sound.

Sabella (Savig.). species various. In the neighbourhood (Rogers).

Spio (Turton). seticornis (Fabr.). In the neighbourhood (Rogers).

Spirorbis (Daudin.). spirorbis (Linn.). Very common.

Serpula (Lin.). vermicularis (Linn.). On old shells. var. tubes solitary. var, tubes clustered. intricata (Linn.).

Aphrodite (Linn.). aculeata (Linn.). Not common.

Lepidonotus (Leach, Mgn.). squamatus (Linn.). Common.

Harmothæ (Kimb.). umbricata (Linn.). Common.

Nereis (Cuv.). brevimana (John.). pelagica (Linn.). Common from low-water mark up to the mud of brackish estuaries. viridis (John.). Common amongst seaweed on rocks in the Sound.

Heteronereis (Oerst.) lobulata (Savig.). longissima (Johnst.).

Glycera (Savig.). dubia (Blain.).

Phyllodoce (Cuv.). viridis (Linn.). Very common in old tubes of Sabella anglica. VOI. I, NO. II. 


\section{ARTHROPODA.}

\section{Crustacea.}

\section{Brachyura.}

Stenorhynchus (Lam.).

phalangium (Penn.). Common. 3-45 fathoms.

tenuirostris (Leach). Common. $6-40$ fathoms.

Achaeus (Leach). cranchii (Leach). Occasional. $6-20$ fathoms.

Inachus (Fabr.).

dorsettensis (Penn.). Occasional. 5-30 fathoms.

dorhynchus (Leach).

leptorinchus (Leach).

Pisa (Leach). gibbsii (Leach).

tetraodon (Leach). Not common. $10-20$ fathoms.

Hyas (Leach). coarctatus (Leach). 40 fathoms.

aranea (L.). Frequent. $6-40$ fathoms.

Maia (Lam.). squinado (Herbst.). Frequent. $3-8$ fathoms.

Eurynome (Leach). aspera (Leach). 4-40 fathoms.

Xantho (Leach). florida (Leach). Rocky coast. Occasional. 6-20 fathoms. ribulosa (Edws.). Occasional. 6-20 fathoms. tuberculata (Conch). Frequent. 4-45 fathoms.

Cancer (Linn.).

pagurus (Linn.). Rocky coast. $0-3$ fathoms.

Pilumnus (Leach). hirtellus (Leach). $\quad 0-3$ fathoms.

Primula (Leach). denticulata (Mont.). Frequent. $4-30$ fathoms.

Carcinus (Leach). mœnas (Linn.). Common in estuaries and creeks. $0-\frac{1}{2}$ fathom.

Portumnus (Leach). variegatus (Leach) (latipes, Penn.).

Portunus (Leach). puber (Linn.). Abundant in crab-pots in September. corrugatus (Leach). Rare in Sound. $0-\frac{1}{2}$ fathom. arcuatus (Leach). depurator (Leach). Occasional. 4-45 fathoms. marmoreus (Leach). Occasional. 3-45 fathoms. holsatus (Fabr.). Occasional. pusillus (Leach). Occasional. 5 fathoms.

Polybius (Leach). henslowii (Leach). Occasional in herring and other nets, or trawled.

Pinnotheres (Latr.). pisum (Penn.). Found in mussel at Saltash. veterum (Box). Found in Pinna. 30 fathoms.

Gonoplax (Leach). angulata (Leach). Not uncommon. 12 fathoms.

Planes (Leach). linnæana (Leach). Rare. Found on living turtle near French coast. 
Ebalia (Leach).

pennantii (Leach). (tuberosa, Penn.) Frequent. 40 fathoms.

bryerii (Leach). Frequent. $4-45$ fathoms.

cranchii (Leach). Sound. Frequent. $40-45$ fathoms.

Atelecyclus (Leach).

heterodon (Leach). Occasional. 45 fathoms.

Corystes (Leach).

cassivelaunus (Leach). Common. 12 fathoms.

Thia (Leach).

polita (Leach).

\section{Anomoura.}

Pagurus (Fabr.).

bernhardus (Linn.). Very common. $0-30$ fathoms.

prideauxii (Leach). Sound. Occasional. 6-45 fathoms.

cuanensis (Thomp.). Off Plymouth. Not common. 3-10 fathoms.

hyndmanni (Thomp.). Near Plymouth. Occasional. 6 fathoms.

lævis (Thomp.). Dredged off Eddystone. Occasional. 4-10 fathoms.

forbesii (Bell).

thompsonii (Bell).

ulidianus (Thomp.).

fasciatus (Bell).

dillwynii (S. Bate). Mouth of Yealm River near Plymouth. Occasionally. 6 fathoms.

Porcellana (Lam.).

platycheles (Penn.). Common. $0-3$ fathoms.

longicornis (Penn.). Common. 4-40 fathoms.

Galathea (Fabr.).

squamifera (Leach.). Occasional. 12 fathoms.

dispersa (S. Bate). Common. 4-40 fathoms.

nexa (Emb.). Occasional. 40 fathoms.

andrewsii (Kinahan). Frequent. $10-45$ fathoms.

bamffica (Penn.). (Rondeletii, Bell.) Common in stomach of cod-fish, or $20-30$ fathoms.

digitidistans (S. Bate). 30 fathoms.

strigosa (Fabr.). Common. 0-10 fathoms.

\section{Macroura.}

Arctus (Fabr.). arctus (Linn.). (Ursus, S. B.) Rare. 6 fathoms.

Palinurus (Fabr.). vulgaris (Latr.). Common. 3-10 fathoms.

Homarus (Linn.). vulgaris (Edw.). (Marinus, Fabr.). Common. $1-6$ fathoms.

Callianassa (Leach). subterranea (Leach). One specimen. 4 fathoms. Rare.

Gebia (Leach). stellata (Mont.). Shores of Sound. deltura (Leach).

Axia (Leach). Rare. stirhynchus (Leach). Near Plymouth.

Crangon (Fabr.). Common. vulgaris (Fabr.). $\quad 0-40$ fathoms. fasciatus (Risso). Occasional. 20 fathoms. spinosus (Leach). Frequent. 6-15 fathoms. sculptus (Bell). 20 fathoms. trispinosus (Hailstone). Rare. 6 fathoms. 
Alpheus (Fabr.).

ruber (Edw.). Not common. 30 fathoms.

affinis (Guise). Not common. 30 fathoms.

Typton (Hellar).

spongiosum (S. Bate). Rare, within sponge. 4 fathoms.

Nika (Risso). edulis (Risso). Occasional. 30 fathoms. Rare.

Athanas (Leach). nitescens (Mont. Leach). Off Polperro.

Hippolyte (Leach).

varians (Leach). In rock-pools, and dredged 6-10 fathoms in Sound. cranchii (Leach). Common.

tenuirostris (S. Bate). Several specimens. $4-6$ fathoms.

bartlui (S. Bate).

spirontocaris (S. Bate) (Hippolyte).

spinus (Leach). 30 fathoms.

Palæmon (Fabr.).

serratus (Penn). Common. $1-40$ fathoms.

squilla (Fabr.).

leahii (Bell). varians (Leach).

Caridion (Goës).

gordoni (Sp. Bate).

\section{Stomatopoda.}

Mysis (Latr.).

chamœleon (Thomp.). 5 fathoms.

vulgaris (Thomp.).

griffithsiæ (Bell).

Thysanopoda (Edw.). couchii (Bell).

Cuma (Edw.). scorpioides (Mont.). unguiculata (S. Bate).

Vaunthomsonia (S. Bate). edwardsii (Kröyer). cristata (S. Bate).

Diastylis (say, Alauna, Goodsir, Bell). rathkii (Kröyer) (rostrata, Goodsir, Bell).

Eudora (S. Bate). truncatula (S. Bate).

Iphithöe (S. Bate) (Halia, S. Bate, White). trispinosa (Goodsir).

Cyrianassa (S. Bate) (Venilia, S. Bate, White). gracilis (S. Bate). longicornis (S. Bate).

Squilla (Fabr.). desmarestii (Risso). Rare.

Phyllosoma (Leach). cranchii (Leach). Surface. Rare.

Talitrus (Latreille).

\section{Amphipoda.}

locusta (Linn.). Abundant in rock-pools between tide-marks.

Orchestia (Leach).

littorea (Mont.). Under Mount Batten.

deshayesii (Savig.). Under Mount Batten. Rare.

mediterranea (Costa) (lævis, S. Bate). 
Allorchestes (Dana). nilssonii (Kröyer) (Danai, S. Bate). Shores of Sound. imbricatus (S. Bate). Holes in Breakwater.

Nicea (Nicolet) (galanthis, S. Bate). lubbockiana (S. Bate).

Montagna (S. Bate). monoculoides (Mont.) (Typhis monoculoides, White, Gosse). marina (S. Bate). alderii ( $\mathrm{S}$. Bate). pollexiana (S. Bate).

Danaia (S. Bate). dubia (S. Bate). Trawled off Plymouth.

Lysianassa (M. Edw.). costæ (M. Edw.). audoniniana (S. Bate). Dredged in Sound. longicornis (Lucas). Dredged in Sound. atlantica (Edw.) (marina, S. Bate). Dredged in Sound.

Anonyx (Kröyer). edwardsii (Kröyer). Dredged in Sound. minutus (Kröyer). Dredged in Sound. holbolli (Kröyer). ampulla (Kröyer). denticulatus (S. Bate). longipes (S. Bate). obesus (S. Bate). longicornis (S. Bate). typica (Kröyer).

Callisoma (Hope). crenata (S. Bate). Near Eddystone.

Ampellisca (Kröyer). gaimardii (Kröyer) (typica, S. Bate). Dredged in Sound. belliana ( $\mathrm{S}$. Bate). In Sound.

Westwoodilla (S. Bate). cæcula (S. Bate). In trawl refuse near Eddystone. hyalina (S. Bate). In trawl refuse near Eddystone.

Kröyera (S. Bate). arenaria (S. Bate).

Phoxus (Kröyer). simplex (S. Bate) (Kröyeri, S. Bate). Dredged in Sound. plumosus (Hoböll). Dredged in Sound. holbölli (Kröyer). Dredged in Sound.

Monoculodes (Stimp.). stimpsoni (S. Bate). Near Plymouth.

Urothöe (Dana). elegans (S. Bate).

Lilgeborgia (S. Bate). pallida (S. Bate). East of Drake's Island.

Isæa (M. Edw.). montagui (M. Edw.). In trawl refuse near Eddystone.

Iphimedia (Rathke). obesa (Rathke). North-west of Drake's Island. eblanæ (S. Bate).

Acanthonotus (Owen). testudo (Mont.).

Dexamine (Leach). loughrinii (S. Bate). 5 fathoms.

Atylus (Leach). bispinosus (S. Bate). Whitsand Bay. huxleyanus (S. Bate). swammerdamii (M. Edw.). In Sound. 
Pherusa (Leach).

fucicola (Edw.). On rocky shores.

Calliope (Leach). lævinscula (Kröyer).

Leucothöe (Leach). articulosa (Mont.). Plymouth Sound.

Lembos (S. Bate). versiculatus (S. Bate). danmoniensis (S. Bate).

Aöra (Kröyer). (Lalaria, Nicolet.) gracilis (S. Bate). In trawl refuse near Eddystone.

Eurystheus (S. Bate). tridentatus (S. Bate). tuberculosus (S. Bate). erythrophthalmus (S. Bate). In Sound.

Gammarella (S. Bate). brevicaudata (M. Edw.). (Orchestiformis, S. Bate.)

Melita (Leach). palmata (Leach). Brackish water. gladiosa (S. Bate). Sound,

Amathia (Rathke). sabinii (Leach). In Sound.

Grammarus (Fabr.).

locusta (Fabr.)

gracilis (Rathke). camptolops (Leach). longimanus (Leach). palmatus (Mont.). (Inæquimanus, S. Bate.) grossimanus (Mont.). maculatus (Johns.). marinus (Leach). Trawled off Plymouth.

Meganæra (S. Bate). semiserrata (S. Bate). Near Mallard Buoy in Sound. brevicaudata (S. Bate).

Bathyporeia (Lindström). (Thersites, S. Bate.) pilosa (Lindst.). pelagica (S. Bate).

Leucothoë (Leach). articulosa (Mont.). In Sound. farina (Savig.). (Procera, S. Bate.)

Microdeutopus (Costa). anomalus (Rath.). In sponge under Hoe. versiculatum (S. Bate). Dredged near Plymouth.

Amphithoë (Leach). rubricator (Mont.). Dredged in Sound. littorina (S. Bate). On shore been tide-marks. gammaroides (S. Bate).

Melita (Leach). obdusata (Leacis) In Sound.

Mœra (Leach). grossimana (Mont.). Frequent in Sound. brevicaudata (S. Bate). Dredged off Plymouth.

Sunamphithöe (S. Bate). conformata (S. Bate).

Podocerus (Leach). falcatus (Mont.). variegatus (Leach). Among confervæ and rock corallines. pulchellus (Leach).

capillatus (Rath.). Trawled off Plymouth. 
Cerapus.

abditus. In Sound.

Nænia (S. Bate). tuberculosa (S. Bate). Dredged off Plymouth.

Jassa (Leach). pelagica (Leach).

Siphonocutus (Kröyer). whitei (Gosse).

Erichthonius (M. Edw.). difformis (M. Edw.).

Cyrtophium (Dana). darwinii (S. Bate).

Corophium (Latreille). longicorne (Fabr.). In Sound. bonellii (M. Edw.).

Chelura (Phillipi). terebraus (Phil.). In Sound.

Hyperia (Latr.). galba (Mont.). (Latreillii, Edw.) fabricii (M. Edw.).

Proto (Leach). pedata (Leach). goodsirii (S. Bate).

Protella (Dana). longispina (Kröyer). (Phasma.)

Caprella (Lam.). linearis (Latr.). pennantii (Leach). tuberculosa (Goodsir).

lobata (Müll.). Near Plymouth. acanthifera (M. Edw.). Drake's Island, low water, and dredged. acutifrons (Latreille). In the neighbourhood. hystrix (Kröyer). In the neighbourhood. æquilibra (S. Bate). In the neigbourhood.

\section{Isopoda.}

Arcturus (Latr.). (Astacilla, Johns.)

leachia (Johns.).

longicornis (Sowerby). Off Plymouth, attached to Echinus.

Anthura (Leach). gracilis (Mont.).

Conilera (Leach). cylindricus (Mont.). Knap Buoy. 6 fathoms.

Tanais (M. Edw.). dulongii (Audouin). hirticaudatus (S. Bate).

Paratanais (Dana). forcipatus (Lillj.). Dredged in Sound.

Apseudes (Leach). talpa (Mont.). Dredged in Sound.

Anceus (Risso). maxillaris (Mont.). $\operatorname{rapax}($ M. Edw.).

Pranixa (Leach). (Tem of Anceus.) ceruleata (Mont.). fusca (Johns.). edwardsii (S. Bate). 
Ione (Mont.).

thoracica (Mont.)

Bopyrus (Latr.). squillarum (Latr.).

Munna (Kröy.). kroyeri (Good). whiteana (S. Bate).

Jaera (Leach). albifrons (Leach). nordmanni Rath.).

Oniscoda (Latr.). maculosa (Leach). deshayesii (Lucas).

Limnoria (Leach). lignorum (Rath.). (Terebrans, Leach.) In wood-work in sea.

Idotea (Fabr.). pelagica (Leach). Eddystone. tricuspidata (Desm.). emarginata (Fabr.).

linearis (Fabr.). Near Plymouth. acuminata (Leach). appendiculata (Risso). parallela (S. Bate).

Ligia (Fabr.). oceanica (Linn.). Abundant on shore.

Sphæroma (Latr.). serratum (Fabr.). Sound. Dredged. rugicauda (Leach).

Dynamene (Leach). rubra (Leach). Near Plymouth.

Cymodocea (Leach). truncata (Leach). emarginata (Leach). Mount Edgcumbe. montagui (Leach). rubra (Leach). viridis (Leach).

Neræa (Leach). bidentata (Adams). Rocky shores.

Campecopea (Leach). hirsuta (Mont.). cranchii (Leach).

Eurydice (Leach). pulchra (Leach).

Ega (Leach). bicarinata (Leach). Trawled in Sound. tridens (Leach).

Cirolana (Leach). cranchii (Leach). Knap buoy. 6 fathoms.

Rocinela (Leach). danmoniensis (Leach). Rare, in Sound.

\section{Ostracoda.*}

Pontocypris. mytiloides (Norman). trigonella (Sars). augusta (Brady).

* The following species were dredged at 40 fathoms in the neighbourhood of the Eddịstone. 
Bairdia.

inflata (Norm.).

acanthigera (Brady).

Cythere.

pellucida (Baird).

tenera (Brady).

ladia (Brady).

convexa (Baird).

fusmaichica (Sars).

villosa (Sars).

emaciata (Brady).

somipunctata (Brady).

cuneiformis (Brady).

antiqua (Baird).

jonesii (Baird).

Eucythere. acerosa (Brady). parva (Brady).

Loxoconcha.

impressa (Baird).

guttata (Norm.).

tamarindus (Jones).

Xestoleberis.

aurantia (Baird).

Cytherura.

angulata (Brady).

cuneata (Brady).

thiata (Sars).

similis (Sars).

acuticosta (Sars),

Cytheropteron.

punctatum (Brady).

nodosum (Brady).

multiforum (Norman).

subcircinatum (Sars).

Bathocythere.

constricta (Sars).

turgida (Sars).

Pseudocythere.

caudata (Sars).

Sclerochilus.

contorus (Norman).

Paradoxostomata. ensiforme (Brady).

Polycope.

abbreviatum (Sars).

compressa (Brady).

\section{Cirripedia.}

Alcipe (Hauc.).

lampas (Hauc.).

Balanus (Auct.).

balanoides (Linn.).

porcatus (Costa).

Pyrgoma (Leach.).

anglicum (Sowerby).

Chthamalus (Ranz.).

stellatus (Ranz.). 
Lepas (Linn.).

hillii (Dar.). anatifera (Linn.).

Scalpellum (Leach).

vulgare (Leach). Attached to Plumularia.

Pygnogonum (Brün).

Pycnogonida.

littorale (Müll). Sound; Drake's Island.

\section{MOLLUSCA.}

Teredo (Sell.).

\section{Lamellibranchiata.}

norvegica (Speng.). In submerged wood in Sound.

navalis (Linn.). In wood piles in rocks.

megotara (Haul.).

var. mionota, Drift wood in Plymouth.

malleolus (Turt.). In Plymouth.

Pholas (Linn.).

dactylus (Linn.). In stones of Breakwater (Bellamy).

striata (Linn.). In mahogany in ship-building yards.

crispata (Linn.). Bellamy records it.

parva (Penn.). Bellamy records it.

Saxicava (F. de Bell.).

arctica (F. \& H.). In limestone rocks, around Sound and at Breakwater.

rugosa (Linn.). In limestone rocks, around Sound and at Breakwater.

Venerupis (Lam.).

irus (Linn.). In crevices of limestone rocks.

Mya (Linn.). -35 fathoms.

arenaria (Linn.). Common all along south coast.

truncata (Linn.). Only one specimen found.

Panopea (M. de la Groye). plicata (Mont.). Trawl refuse off Plymouth.

Corbula (Brug.). 20-180 fathoms.

gibba (Oliv.). Probably in Sound.

Lyonsia (Turt.). 5-80 fathoms. norvegica (Chaun.). Probably in or near Sound.

Thracia (Leach). $30-100$ fathoms.

pubescens (Pult.). In Plymouth Sound.

convexa (Wood). Probably in the Sound.

papyracea (Poli.). In the Sound.

praetenuis (Pult.). Probably near Sound.

Solen (Linn.). $\quad 0-8$ fathoms.

siliqua (Linn.). Abundant in Whitsand Bay, near Raeme Head. vagina (Linn.). Probably in deep sand, Whitsand Bay.

ensis (Linn.). In Whitsand Bay.

pellucidus (Penn.). Probably in Plymouth Sound.

Solecurtis (de Blain). 15-25 fathoms.

antiquatus (Pult.). Rare in Sound. 25 fathoms. candidus (Renier). Rare. Probably in Sound.

Ceratisolen (Forbes). 15-25 fathoms. legumen (Linn.). Probably in Sound.

Psammobia (Lamk.).

vespertina (Chem.). Probably in Sound. Muddy sand at low water. ferröensis (Chem.). Probably in Sound. Muddy sand at low water. tellinella (Lamk.). In the Sound. 25 fathoms.

costulata (Turt.). Probably in the Sound in deep water. 
Gastrana (Schum.).

fragilis (Linn.). Probably in the Sound.

Tellina (Linn.). crassa (Gmel.). In the Sound. Dredged.

donacina (Linn.). Probably in the Sound in 20 fathoms.

pusilla (Phil.). Whitsand Bay, near Raeme Head. 3-85 fathoms.

tenuis (da Costa). Whitsand Bay.

fabula (Gron.). Whitsand Bay.

balthica (Linn.). In the Sound.

balaustina (Linn.). In trawl refuse from 20 fathoms off the coast.

Scrobicularia (Schum.). Low-water to 4 fathoms.

piperata (Bellon.). Common in sandy mud. Probably in estuaries of Plym and Tamar.

alba (Wood). Whitsand Bay.

prismatica (Mont.). Whitsand Bay.

Donax (Linn.). Littoral or sublittoral. trunculus (Linn.). Whitsand Bay.

politus (Poli). Whitsand Bay.

vittatus (Da Costa). Common all along coast (Parfitt).

Amphidesma (Lam.). 20 fathoms.

castaneum (Mont.). Probably in Sound. Rare.

Mactra (Linn.). Low-water to 50 fathoms.

solida (Linn.). In the Sound and Whitsand Bay.

var. truncata (Mont.). In the Sound.

var. elliptica (Brown). In the Sound.

stultorum (Linn.). Whitsand Bay. Abundant.

subtruncata (da Costa). In the Sound.

Lutraria (Lamk.). Low-water to 25 fathoms.

elliptica (Lamk.). Whitsand Bay.

oblongata (Chem.). Probably in Sound or Whitsand Bay. Rare.

Tapes (Mühlf.). Littoral to 140 fathoms.

aureus (Gmel). Off Plymouth. 3-10 fathoms.

virgineus (Linn.). In trawl refuse. 5-35 fathoms.

pullastra (Mont.). In the Sound. $0-7$ fathoms.

var. perforans. In limestone in the Sound.

Venus (Linn.).

(Linn.). Probably in Sound at low-water mark.

verucosa (Linn.). Probably in Sound. Littoral to 7 fathoms.

casina (Linn.). Probably in Sound. 12-145 fathoms.

var. reflexa (Mont.). Probably in Sound.

gallina (Linn.). In the Sound. Common 0-100 fathoms.

fasciata (Da Costa). In the Sound. Littoral to 60 fathoms.

ovata (Penn.). In the Sound. 3-100 fathoms.

chione (Linn.). In trawl refuse. Not uncommon.

exoleta (Linn.). Probably in Sound.

lincta (Pult.). Common in Sound.

Circe (Schum.).

minima (Mont.). Probably in Sound.

Lucinopsis (Forb. and Han.). 3-80 fathoms. undata (Penn.). Whitsand Bay. Not common.

Cyprina (Lamk.). islandica (Linn.). Probably in Sound.

Astarte (Sower.).

sulcata (da Costa). Dredged in the Sound and Whitsand Bay. $8-80$

fathoms.

var. elliptica (F. and H.). In the neighbourhood (Rogers).

triangularis (Mont.). Dredged in the Sound. 5-55 fathoms.

Isocardia (Lam.).

cor (Linn.). In trawl refuse from deep water. 
Cardium (Linn.). Littoral or sublittoral in general.

aculeatum (Linn.). In Plymouth Sound in deep water. Rare.

echinatum (Linn.). In the Sound in deep water. Common.

edule (Linn.). In sand or sandy mud. Estuaries of Plym and Tamar. Common. nodosum (Turt.). Whitsand Bay, near Raeme Head. 3-80 fathoms.

fasciatum (Mont.). Whitsand Bay. 5-50 fathoms.

norvegicum (Speng.). Probably in Sound. 15-30 fathoms.

tuberculatum (Linn.). In deep water (Bellamy).

exiguum (Gmel.). Oozy ground. Probably in Sound. 13-15 fathoms.

Lucina (Brug.).

borealis (Linn.). Probably in the Sound. Low-water to 90 fathoms.

spinifera (Mont.). In the Sound. 28 fathoms.

Axinus (Sowby.)

flexuosus (Mont.). Probably in the Sound.

Loripes (Poli.).

lacteus (Linn.). Probably in the Sound.

Diplodonta (Brown).

rotundata (Mont.). Found dead in Sound in 22 fathoms.

Cyamium (Phil.).

minutum (O. Fabr.). Whitsand Bay. Between tide-marks.

Kellia (Turt.). Low-water to 60 fathoms.

suborbicularis (Mont.). Probably in Sound.

Lasæa (Leach). rubra (Mont.). Whitsand Bay.

Lepton (Turt.). squamosum (Mont.). In the Sound in 22 fathoms. clarkiæ (Clark). In the Sound. 18-80 fathoms.

Galeomma (Turt.). turtoni (Ed. of 'Zool. Journl.'). Probably in Sound. 3-4 fathoms.

Mytilus (Linn.).

edulis (Linn.). In the Sound and estuaries. Abundant. Generally littoral. modiolus (Linn.). In the Sound.

adriaticus (Lam.). Probably in the Sound.

barbatus (Linn.). Probably in the Sound. Rare.

Modiolaria (Beck).

discors (Linn.). On roots of laminaria (Parfitt).

marmorata (Forbes). Probably in Sound, embedded in Tunicata.

costulata (Risso). Prohably in Sound, low-water mark. Rare.

Crenella (Brown).

rhombea (Berk.). Probably in Sound. 20 fathoms. Rare.

Nucula (Lam.).

nucleus (Linn.). Probably in Sound, 7-90 fathoms.

var. radiata (F. \& H.). Probably in Sound. $7-25$ fathoms.

Arca (Linn.).

tetragona (Poli.). In crevices in rocks. Hamoaze.

lactea (Linn.). Whitsand Bay. $7-27$ fathoms.

Pectunculus (Lam.). glycymeris (Linn.). In Sound, 15-25 fathoms.

Avicula (Klein). hirundo (Linn.). In trawl refuse from offing.

Pinna (Lister). rudis (Linn.). In trawl refuse from deep water.

Lima (Brug.). subauriculata (Mont.). In Sound. 25 fathoms. Whitsand Bay. 15-50 fathoms. Rare.

loscombii (Sowerby). Probably in Sound. Low-water to 50 fathoms. hians (Gmelin). Probably in Sound. Low-water to 50 fathoms.

Ostrea (Linn.). edulis (Linn.). Cattewater. Prince Rock. Hamoaze. 
Pecten (Pliny).

varius (Linn.). Probably in Sound. 3-35 fathoms.

pusio (Linn.). Probably in Sound. Low-water to 90 fathoms.

tigrinus (Müll.). From stomachs of flat-fish in Sound. $12-60$ fathoms.

similis (Laskey). In Sound. 2-60 fathoms.

maximus (Linn.). In Sound.

opercularis (Linn.). In Sound.

var. lineata. In Sound.

var. tumida. In Sound.

Anomia (Linn.). Low-water to 30 and up to 100 fathoms.

ephippium (Linn.). In the Sound. Free or attached to Pinna.

var. aculeata. In the Sound. Attached to corallines.

patelliformis (Linn.). 15 fathoms.

Dentalium (Linn.).

$$
\text { Scaphopoda. }
$$

entalis (Linn.). In Sound, and from hake's stomachs.

tarentinum (Lamk.). In Sound, 12-15 fathoms, and 5 to 6 miles from land $7-25$ fathoms.

Chiton (Linn.).

\section{Gasteropoda.}

banleyi (Bean). In trawl refuse.

fascicularis (Linn.). Whitsand Bay. Littoral to 25 fathoms in deep water (Bellamy).

ruber (Linn.). Probably in the Sound.

cinereus (Linn.). In Millbay, low spring tide.

cancellatus (Sow.). Probably in Sound.

marginatus (Penn.). Doubtless in Sound.

Patella (List.). Littoral and laminarian zones.

vulgata (Linn.). In the Sound. "Rock limpet."

var. 1, elevata. In Sound.

var. 2, picta. In Sound.

var. 3 , intermedia. In Sound.

var. 4, depressa (Penn.). In Sound.

var. 5, cerulea (Linn.). In Sound.

Helcion (De Mont.).

pellucidum (Linn.).

var. lœvis. In Sound.

Tectura (Cuv.).

virginea (Müll.). In Sound. " Sea-weed limpet."

Calyptræa (Lamk.).

chinensis (Linn.). In Sound. 7-10 fathoms.

Fissurella (Brug.).

græca (Linn.). Probably in Sound.

Emarginula (Lamk.).

fissura (Linn.). In Sound, in deep water.

rosea (Bell). In Sound. 20-25 fathoms.

Haliotis (Linn.).

tuberculata (Linn.). In the neighbourhood (Rogers).

Trochus (Rond.).

zizyphinus (Linn.). Common in Sound.

var. 1. Common in Sound.

var. 2. Common in Sound.

var. 3. Common in Sound.

granulatus (Born). In Sound.

exasperatus (Penn.). Probably in Sound.

millegranus (Phil.). Probably in Sound. 
striatus (Linn.). Probably in Sound.

montacuti (Wood). Probably in Sound.

tumidus (Mont.). In Sound. Coralline zone. 7-80 fathoms.

cinerarius (Linn.). In Sound. Abundant. Sub-littoral.

umbilicatus (Mont.). In Sound. Abundant. Sub-littoral.

magus (Linn.). Probably in Sound. 10-15 fathoms.

lineatus (Da Costa). Probably in Sound. Between tide-marks.

Cyclostrema (Marr.). Probably in Sound. 10-40 fathoms.

cutlerianum (Clark). Probably in Sound. Found both east and west of Plymouth. serpuloïdes (Mont.). Probably in Sound.

Phasianella (Lamk.).

pulla (Linn.). In Sound and Whitsand Bay.

Ianthina (Bolt.).

rotundata (Leach). Whitsand Bay.

exigua (Lamk.). Cast up in Whitsand Bay during storms. Not British.

Crepidula (Lamk.).

plana (Say). Dredged in the Sound.

Truncatella (Risso).

truncatula (Drap.). Under stones between tide-marks in Sound.

Rissoa (Frém.).

costata (Adams). Whitsand Bay.

parva (Da Costa). Whitsand Bay.

fulgida (Adams). Whitsand Bay.

reticulata (Mont.). On south coast, Devon.

soluta (Phil.). In Sound.

cingillus (Mont.). In Sound.

calathus (F. and H.). Whitsand Bay.

punctura (Mont.). Whitsand Bay.

inconspicua (Ald.). Trawl refuse in Plymouth.

membranacea (Adams). Plentiful on south coast, Devon.

proxima (Ald.). In sound.

vitrea (Mont.). Whitsand Bay.

violacea (Desm.). Probably in Sound.

striata (Adams). Probably in Sound.

Barleeia (Clark).

rubra (Mont.). Whitsand Bay.

Hydrobia.

ulvæ (Penn.). Doubtless in estuaries of Sound.

Lacuna (Turt).

pallidula (Da Costa). On oar-stone.

divaricata (Fabr.). Probably in Sound.

puteolus (Turt). Probably in Sound.

Skenea (Flem.).

planorbis (Fabr.). Doubtless in Sound.

Littorina (Feruss.).

litorea (Linn.). In the Sound and its estuaries.

rudis (Mat.). In the Sound and its estuaries.

var. tenebrosa. Probably in the Sound.

var. patula. On Eddystone Rocks.

obtusata (Linn.). In the Sound and its estuaries.

neritoides (Linn.). In the Sound and its estuaries.

Scalaria (Lamk.).

communis (Lamk.). In the Sound and Hamoaze.

clathratula (Adams). On roots of Corallina officinalis.

trevelyana (Leach). in the Sound.

turtonæ (Turton). Coralline zone.

Cæcum (Flem.).

trachea (Mont.). Probably off Sound. Coralline zone.

glabrum (Mont.). Probably off Sound. Coralline zone. 
Turritella (Lamk.).

terel)ra (Linn.).

var. nivea. In Mill Bay. Low spring tide.

var. gracilis. In Mill Bay. Low spring tide.

Aclis (Lovén).

ascaris (Turt.). In Sound.

supranitida (Wood). In Sound.

gulsonæ (Clark). Probably in Sound.

unica (Mont.) Probably in Sound.

Aporrhais (Da Costa).

pes-pelecani (Linn.). Shores of Mount Edgcumbe. Low-water.

Cerithium (Adan.).

reticulatum (Da Costa). Probably in Sound.

perversum (Linn.). Probably in Sound.

Cerithiopsis (F. and H.).

pulchella (Jeff.). Coraline zone. Sound.

tubercularis (Mont.). Probably in Sound.

barleei (Jeff.). Among trawl refuse. Plymouth.

Stilifer (Brod.).

turtoni (Brod.). Attached to Echinus miliaris (Stewart).

Eulima (Risso).

polita (Linn.). Probably in Sound.

intermedia (Cantr.). Off Plymouth.

distorta (Deshay.). Probably in Sound.

subulata (Don.). Probably in Sound. Coralline zone.

Odostomia (Flem.).

truncatula (Jeff.). In trawl refuse from off Plymouth.

clavula (Lov.). Rare. Dredged Plymouth. 6-50 fathoms.

lukisi (Jeff.). Among weeds at low-water.

pallida (Mont.). In trawl refuse from off Plymouth.

conoïdea (Brocchi). Probably in Sound.

acuta (Jeff.). Coralline zone, probably in Sound.

rufa (Phil.). In trawl refuse off Plymouth.

lactea (Linn.).

insculpta (Mont.). Sand of coralline zones.

interstincta (Mont.). In Bigbury Bay.

decussata (Mont.). Probably in Sound. In coralline zone.

excavata (Phil.). Probably in Sound.

fenestrata (Forbes). Probably in Sound. Muddy ground. 7-12 fathoms.

plicata (Mont.). Probably in Sound.

rissoïdes (Hanl.). Probably in Sound.

scalaris (Phil.). Probably in Sound.

unidentata (Mont.). Probably in Sound.

Otina (Gray.).

otis (Turt.). On rocks under Hoe.

Lamellaria (Mont.).

perspicua (Linn.). Dredged in Sound.

Natica (Adan.).

catena (Da Costa). Whitsand Bay.

alderi (Forbes). Probably in Whitsand Bay.

Adeorbis (S. Wood).

subcarinatus (Mont.). Whitsand Bay. In 12 fathoms. montacuti (Forbes). In Sound.

Cypræa (Linn.).

europæa (Mont). Common.

Ovula (Brug.). patula (Penn.). Probably in Sound.

Marginella (Lamk.).

lævis (Don.). Probably in Sound. 
Defrancia (Mill.).

leufroyi (Mich.). Probably in Sound.

teres (Forbes). Dredged. 15-85 fathoms.

gracilis (Mont.). Not uncommon on coast in coralline zone.

linearis (Mont.). Common on coast in laminarian and coralline zones and deep water.

reticulata (Ren.). In Sound. Coralline zone.

purpurea (Mont.). Doubtless at mouth of Sound.

Pleurotoma (Lamk.).

attenuata (Mont.). On coast. Coralline zone.

costata (Don.). Low-water mark in pools in Sound.

brachystoma (Phil.). On muddy sand. 10-60 fathoms.

nebula (Mont.). Common in sand, low-water.

var. elongata. In deep water.

rufa (Mont.).

var. cranchii.

striolata (Phil.). Probably off Sound.

Purpura (Brug.).

lapillus (Linn.). Common in Sound.

var. several.

Buccinum (Linn.).

undatum (Linn.). Common in Sound and estuaries.

Nassa (Lamk.).

reticulata (Linn.). In the Sound between tide-marks. Common.

incrassata (Ström.). Dredged frequently in Sound.

pygmæa (Lamk.). Coralline zone, probably in Sound.

Murex (Linn.).

erinaceus (Linn.). In the Sound.

aciculatus (Lamk.). Dredged in coralline zone. Common.

Trophon (de Mont.).

muricatus (Mont.). Muddy sand, coralline zone. On coast.

Fusus (Brug.).

antiquus (Linn.). Perhaps in Sound.

buccinatus (Lamk.). Dredged in deep water.

gracilis (da Costa). In the Sound.

Cylichna (Lov.).

\section{Pleurobranchia.}

acuminata (Brug.). In trawl refuse. Plymouth.

umbilicata (Mont.). Probably near Plymouth.

cylindracea (Penn.). Probably near Plymouth.

Utriculus (Brown). Laminarian zone. mammillatus (Phil.). Probably near Plymouth. trunculatus (Brug.). Doubtless at Plymouth.

Actæon (de Mont.). tornatilis (Linn.). Whitsand Bay.

Bulla (Klein). hydatis (Linn.). In Sound.

utriculus (Broc.). In Sound. Muddy sand.

Scaphander (De Mont.). lignarius (Linn.). In the Sound.

Philine (Ascanius).

catena (Mont.). Probably in Sound.

punctata (Clark). Whitsand Bay.

pruinosa (Clark). Dredged off Plymouth.

aperta (Linn.). Probably Whitsand Bay

Aplysia (Linn.).

punctata (Cuv.). Whitsand Bay. On rocks.

depilans (Linn.). Mouth of Sound. 5 fathoms. 
Pleurobranchus (Cuv.).

membranaceus (Mont.). In the Sound.

plumula (Mont.). In the Sound.

\section{Nudibranchia.}

Elysia (Risso). viridis (Mont.). In the neighbourhood (Rogers).

Eolis (Cuv.). papillosa (Linn.). Low-water Cremill. coronata (Forbes). In the neighbourhood (Rogers). rufibranchialis (John.). Trawled 10 miles south-east of Plymouth. Polycera (Cuv.). quadrilineata (Müll.). Tide pools and Yam Gut. 4 fathoms.

Goniodoris (Forbes). nodosa (Mont.). Fairly frequent at low-water. Under the Hoe.

Doris (Linn.). tuberculata (Cuv.). Fairly common in Sound. coccinea (Forbes). On shore and trawled in Sound. testudinaria (Risso). Trawled in Sound.

Dendronotus (A. and Han). arborescens (Müll.). Off coast.

Doto (Oken). coronata (Gmel). 5 miles south of Eddystone. 35 fathoms.

Limapontia (John.). nigra (John.). On shore of Sound.

Oncidium (Buchanan).

\section{Pulmonata.} celticum (Cuv.). Whitsand Bay. On rocks about high-water mark.

Spirialis (Eyd. and Soul.).

\section{Pteropoda.}

retroversus (Flem.). On the coast.

Ommatostrephes (D'Orb.).

\section{Cephalopoda.} sagittatus (Lamk.). On the coast.

Loligo (Schn.). vulgaris (Lamk.). Common in Sound and off coast.

Sepiola (Rond.). rondeleti (Leach). In the neighbourhood (Rogers).

Sepia (Pliny). officinalis (Linn.). Off the coast and in the Sound. biserialis (De Mont.). Off the coast.

Octopus (Lamk.). vulgaris (Lamk.). Along the coast.

Eledone (Leach). cirrosa (Lamk.). In the neighbourhood (Rogers).

\section{BRACHIOPODA.}

\section{Testicardinea.}

Terebratula (Lhwyd.).

caput-serpentis (Linn.). In neighbourhood (Rogers).

Argiope (Deslong.).

capsula (Jeff.). Off Plymouth. 18-25 fathoms.

VOL. I, NO. II. 
Pedicellina (Sars).

POLYZOA.

$$
\text { Entoprocta. }
$$

cernua (Pallas). In tide pools on shores of Sound.

gracilis (Sars). Common between tide-marks on coast.

Crisia (Lamour.).

$$
\text { Ectoprocta. }
$$

eburnea (Linn.). On roots of laminaria all along coast.

denticulata (Lam.). On roots of laminaria all along coast.

cornuta (Linn.). On surface rocks along coast.

Diastopora (Lamour.).

obelia (Johns.). On shells from deep water.

suborbicularis (Hincks). On stones, \&c. Shallow to deep water. patina (Lam.). On shells and stones along coast.

Lichenopora (Defr.).

hispida (Flem.). On shells and stones from deep water.

Stomatopora (Bronn).

deflexa (Couch). Common off Deadman.

fungia (Couch). Common from Eddystone to Deadman.

Tubulipora (Lamk.).

flabellaris (Fabr.). On Pecten maximus shells.

Idmonea (Lamx.).

serpens (Linn.). On shells, \&c., all along coast.

Alcyonidium (Lamx.).

gelatinosum (Linn.). Common, low-water mark on coast.

hirsutum (Flenı.). On shells, low-water mark. mytili (Dalyell). On stones between tide-marks.

Flustrella (Gray).

hispida (Fabr.). Common on Fucus serratus.

Vesicularia (Thomp.). spinosa (Linn.). Off the Deadman. Rare.

Amathia (Lamx.). lendigera (Linn.). On algæ.

Buskia (Alder). nitens (Alder). Obtained from the Sound.

Valkeria (Flem.) uva (Linn.). In shallow water, on fuci, \&c.

Mimosella (Hincks). gracilis (Hincks). On Halidrys siliquosa,

Scrupocellaria (v. Beneden). scruposa (Linn.). Very common.

reptans (Linn.). Roots of large algæ on coast.

Eucratea (Lamx.). chelata (Linn.). Common on large algæ.

Atea (Lamx.). anguina (Linn.). Very abundant, on algæ. truncata (Landsb.). On shells. Not common.

Bicellaria (Blainv.). ciliata (Linn.). Roots of large algæ on coast. Trawled near Eddystone.

Bugula (Oken).

turbinata (Alder). Rocks near low-water mark.

flabellata (Thomp.). Common. From moderate to deep water. avicularia (Linn.). Roots of laminaria. Not common. calathus (Norm.). Off the coast.

Cellaria (Lamx.). fistulosa (Linn.). Eddystone and thereabouts. sinuosa (Hassal). Eddystone and eastward. 
Flustra (Linn.).

follacea (linn.). Washed ashore.

papyracea (E. and Sol.). Shallow water on coast.

Membranipora (Blainv.).

lacroixii (Aud.). Frequent.

catenularia (Jameson). Most common.

pilosa (Linn.). Very common.

membranacea (Linn.). On algæ. Abundant.

lineata (Linn.). Common between tide-marks to deep water. On weed, stone, \&c.

flustroides (Hincks). Off Deadman. 60 fathoms.

dumerilii (Aud.). Abundant on shells, \&c. Shallow water.

imbellis (Hincks). Off coast, east and west of Sound. 60 fathoms.

flemingii (Busk). Common on shells, \&c. Shallow to deep water. nodulosa (Hincks). Off Brixham.

Cribrilina (Gray). punctata (Hassall). Between tide-marks.

Membraniporella (Smitt).

nitida (Johns.). Common on stones between tide-marks.

Microporella (Hincks).

ciliata (Pallas). Common, shallow and deep water.

malusii (Aud.). On the neighbouring coast.

impressa (Aud.). Off Deadman and coast to eastward.

violacea (Johns.). Off coast.

Chorizopora (Hincks).

brongniartii (Aud.). Abundant on shells, \&c., in shallıw w: ter.

Schizoporella (Hincks).

unicornis (Johns.). On stones hetween tide-marks. Common.

spinifera (Johns.). Ruots of large laminaria.

linearis (Hassal). On coast. Abundant.

hyalina (Linn.) Not scarce on coast.

Mastigophora (Hincks).

dutertrei (Aud.) Off Deadman. 60 fathoms.

Schizotheca (Hincks). fissa (Busk). On coast and off Deadman.

Lepralia (Johns.).

pallasiana (Moll.). Abundant between tide-marhs.

foliacea (E. and Sol.). On coast and near Eddistone. Cummon.

pertusa (Esper). On coast. Common off Deadman.

edax (Busk.). In the Sound on Turitella.

Por ella (Gray).

concinna (Busk). Off Deadman.

Smittia (Hincks).

affinis (Hincks). Start Bay on a shell.

trispinusa (Johns.). Very common shallow to deep water.

Phỵlactella (Hincks).

eximia (Hincks). Off Deadman.

Mucronella (Hincks).

variolosa (Johns.). Off Deadman. 60 fathoms. coccinea (Abild.). Common littoral.

Palmicellaria (Alder), skenei (E. and Sol.). Off Deadman. Rare.

Rhyncopora (Hincks.).

bispinosq ('ohns.). Off Deadman. 60 fathoms.

Cellepora (Fabr.).

pumicosa (Linn.). Common, encrusting old shells, \&c.

avicularis (Hincks). Common on Sertularians at moderate to great depths. costazii (Aud.). On Anomia. 


\section{TUNICATA.}

Ascidia (Linn.).

\section{Ascidix simplices.}

intestinalis (Linn.) Common in Sound.

virginea (O. F. Miill.). Not abundant in Sound. aspera (O. F. Müll.). Not abundant in Sound. vitrea.? In the neighbourhood (Rogers).

Molgula (Forb.). occulata (Kupff). Dredged in Sound.

Perophora (Wiegm.). listeri (Wiegm.). Abundant.

Cynthia (Sav.).

? On rocks in plenty. quadrangularis. ? In the neighbourhood (Rogers).

Clavelina (Sav.).

lepadiformis (0. F. Müll.). In the neighbourhood (Rogers).

Polycyclus.

Ascidiæ compositæ.

savignyi (Herdm.).

Botryllus (Gärtn.). Everywhere. violaceus (M. Edwards). rubrum (M. Edwards).

Botrylloides (Edw.). Everywhere.

Leptoclinum (Edw.). On shores of Sound.

Distoma (Gärtn.). Very common.

Polyclinum (Sav.). Very common.

\section{PISCES.}

\section{Cyclostomata.}

Petromyzon (Artedi).

marinus (Linn.).

fluviatilis (Linn.). Mud of Sound and rivers.

\section{Chondropterygir.}

Accipenser (Artedi).

\section{Ganoidei.}

sturio (Linn.). Sturgeon. A few caught each year.

\section{Elasmobranchii.}

Carcharias (Müll. and H.). glaucus (Cuv.). Blue shark. Taken in the Sound.

Mustelus (Cuv.). vulgaris (Müll. and H.). Smooth, hound. Frequent.

Galeus (Cuv.). vulgaris (Flem.). Tope. Common.

Lamna (Cuv.). cornubica (Cuv.). Porbeagle. In drift nęts occasionally.

Alopias (Rafin.). vulpes (Bonap.). Thrasher. Off the coast, occasional.

Selache (Cuv.). maxima (Cuv.). Basking shark. Off the coast occasionally. 
Scyllium (Cuv.).

canicula (Cuv.). Small-spotted dog-fish. Common.

catulus (Cuv.). Nurse-hound. Common.

Acanthias (Risso).

vulgaris (Risso). Picked dog-fish. Very common.

Echinorhinus (Blainv.). spinosus (Blainv.). Spinous shark. Taken off Plymouth.

Rhina (Klein). squatina (Raf.). Monk-fish. Common in deep water and in Sound.

Torpedo (Duméril).

Raia (Art.). nobiliana (Bonap.). Torpedo. Rare, taken in Sound.

batis (Linn.). Skate. Very common.

alba (Lacép.). Bordered ray. Occasional. circularis (Couch.) Sandy ray. Common.

clavata (Linn.). Thorn-back ray. Very common. maculata (Mont.). S iotted ray. Common.

macrorhynchus (Raf.). Flapper-skate. Has been obtained from Plymouth.

Trigon (Adanson).

pastinaca (Cuv.). Sting-ray. Taken in the Sound.

Myliobatis (Cuv.).

aquila (Cuv.). Eagle-ray. Rare.

\section{TELEOSTEI.}

\section{Acanthopterygii.}

Labrax (Cuv.). lupus (Cuv.). Bass.

Serranus (Cuv.). cabrilla (Cuv.). Comber. In estuaries. Not common.

Polyprion (Cuv.). cernium (Val.). Stone-bass. Not uncommon off coast.

Mullus (Linn.). barbatus (Linn.). Red mullet. Common. surmuletus (Linn.). Surmullet. Estuaries. Not common.

Cantharus (Cuv. and Val.). lineatus (Thomp.). Bream, or old-wife. Common.

Box (Cuv. and Val.). vulgaris (Cuv, and Val.). Bogue. One recorded from near Plymouth.

Pagellus (Cuv, and Val.). erythrinus (Cuv. and Val.). King of the breams. centrodontus (Cuv. and Val.). Common sea-bream. Especially common. bogaraveo (Cuv.). Spanish bream. Not common.

Trigla (Artedi). cuculus (Linn.). Red gurnard. Common. obscura (Linn.). Lanthorn gurnard. lyra (Linn.). Piper. lineata (Ginel.). Streaked gurnard. gurnardus (Linn.). Gray gurnard. In estuaries. Common hirundo (Linn.). Tub. Common.

Cottus (Artedi). scorpius (Linn.). Father lasher. Common.

bubalis (Euphr.). Lucky proach. Very common on rocky coast, common

Agonus (Bloch, Schneider). also in estuaries.

cataphractus (Bl, schn.). Armed bullhead. Common in estuaries. 
Peristethus (Kaup).

cataphractum (Kaup.). Armed gurnard. One taken in trawl between Plymouth and Eddystone.

Lophius (Artedi).

piscatorius (Linn.). Angler. Frequent. Reach very large size.

Trachinus (Cuv.).

draco (Linn.). Great weaver. All along coast and in offing.

vipera (Cuv. and Val.). Little weaver. All along coast; not common in estuaries.

Scomber (Artedi).

scomber (Linn.). Mackerel. Regular visitors.

Orcynus (Lütken).

thynnus (Lütken). Short-finned tunny. One specimen recorded.

Thynnus (Lütken). pelamys (Cuv. and Val.). Bonito. One taken in Catte Water.

Centrolophus (Lacépède). pompilus (Cuv, and Val.). Black fish. Has been taken off Penlee Point.

Caranx (Lacép.). trachurus (Lacép.). Scad. Regular visitor.

Naucrates (Cuv.). ductor (Cuv and Val.). Pilot fish. Two specimens recorded.

Capros (Lacép.). aper (Lacép.). Cuckoo-fish, Common.

Zeus (Cuv.). faber (Linn.). John Dory. Common.

Xiphias (Artedi). gladius (Linn.). Swordfish. One specimen caught in drift net.

Sciæna (Cuv.). aquila (Risso). Sciæna or Maigre. Off the Coast.

Trichiurus (Linn.). lepturus (Linn.). Silvery hair tail. Caught in herring nets occasionally.

Gobius (Artedi). niger (Linn.). Rock goby. Common. ruthensparri (Euph.). Two-spotted goby. Abundant in Sound. minutus (Gmel.). One-spotted gohy. Common in estuaries.

Callionymus (Linn.). lyra (Linn.). Yellow skulpin. Female plentiful in estuaries, male only in mid-channel.

Cyclopterus (Linn.).

lumpus (Linn.). Lump-fish. Fairly common.

Liparis (Artedi).

vulgaris (Flem.). Sea snail. Common. montagui (Cuv.). Network sucker. Probably in estuaries of Sound.

Lepadogaster (Gouan). gouanii (Lacép.). Cornish sucker. In Sound. Not common. bimaculatus (Flem.). Doubly-spotted sucker. Not common.

Anarrhichas (Artedi). lupus (Linn.). Wolf fish. Rare.

Blennius (Artedi). ocellaris (Linn.). Butterfly blenny. One specimen recorded. gattorugine (Bloch). Tompot. In Sound. Not common. pholis (Linn.). Shanny. Common on rocky shores. galerita (Linn). Montague's Blenny. In estuaries. Uncommon.

Centronotus (Bloch, Schn.). gunnellus (Bloch, Schn.). Butter-fish. Abundant in Sound.

Zoarces (Cuv.). viviparus (Cuv.). Viviparous blenny. In the neighbourhood (Rogers).

Cepola (Linn.). rubescens (Linn.). Red band fish. Taken in Sound. 
Mugil (Artedi).

capito (Cuv.). Gray mullet. Abundant in estuaries.

chelo (Cuv.). Lesser grey mullet. Abundant in estuaries.

Atherina (Artedi). presbyter (Jenyns). Atherine. In the neighbourhood (Rogers).

Gasterosteus (Artedi). aculeatus (Linn.).

var. trachurus (Cuv. and Val.). Rough-tailed stickleback. Common. spinachia (Linn.). Fifteen-spined stickleback. Common in Sound.

Ctenolabrus (Cuv, and Val.). rupestris (Cuv. and Val.). Jago's goldsinny.

Centrolabrus (Günth.). exoletus (Günth). Rock-cook. Abundant in Sound.

Acantholabrus (Cuv, and Val.). palloni (Cuv. and Pal.). Scale-rayed wrasse. A specimen taken off Deadman.

Coris (Lacép.). julis (Günth). Rainbow wrasse.

Labrus (Artedi).

maculatus (Bl.). Ballan wrasse. Abundant in Sound.

mixtus (Fr. o. Ek.). Cuckoo wrasse. Not common in Sound.

lineatus (Don.). Green wrasse.

Crenilabrus (Cuv.).

melops (Cuv.). Cork wing. Abundant in Sound.

cornubicus (Risso). Goldsinny. Abundant in Sound.

Gadus (Cuv.).

\section{Anacanthini.}

morhua (Linn.). Cod. Fairly common.

æglefinus (Linn.). Haddock. Frequent.

luscus (Linn.). Bib. Very common.

merlangus (Linn.). Whiting. Very common.

minutus (Linn.). Poor-cod. Very common.

pollachius (Linn.). Pollack. Very common.

virens (Linn.). Coal-fish, Very common.

Merluccius (Cuv.).

vulgaris (Cuv.). Hake. Common.

Molva (Nil.).

vulgaris (Flem.). Ling. Common.

Motella (Cuv.).

tricirrata (Nil.). Three-bearded rockling. Abundant in estuaries.

cimbria (Nil.). Four-bearded rockling. In the neighbourhood (Rogers).

Raniceps (Cuv.). mustela (Nil.). Five-bearded rockling. In the neighbourhood (Rogers).

raninus (Coll.) Lesser fork-beard. In the Sound.

Ammodytes (Artedi).

launceolatus (Lesauv.). Larger sand launce. Common.

tobianus (Linn.). Lesser sand launce. Common.

Hippoglossus (Cuv.).

vulgaris (Flem.). Holibut.

Rhombus (Cuv.).

maximus (Cuv). Turbot. Fairly common.

lævis (Rondel). Brill. Frequent.

Zeugopterus (Gottsche).

punctatus (Collett). Müller's topknot. Common in estuaries.

unimaculatus (Risso). Bloch's topknot.

Arnoglossus (Bleeker).

megastoma. (?) Merry sole. Common.

laterna (Günth.). Megrim. Common. 
Pleuronectes (Artedi).

platessa (Linn.). Plaice. Very common.

flesus (Linn.). Flounder. Very common.

limanda (Linn.). Dab. Very common.

cynoglossus (Linn.). Craig-fluke.

Solea (Cuv.).

microcephalus (Don.). Smear-dab. Fairly common.

vulgaris (Quensel). Sole. Common.

variegata (Flem.). Thick back. Common in deep water.

lascaris (Risso). Lemon sole. Common.

lutea (Bonap.). Red sole. Taken off coast.

\section{Physostomi.}

Salmo (Artedi).

cambricus (Don.). Salmon trout. Along the coast.

fario (Linn.). Trout. Near to the mouth of the Plym.

Belone (Cuv.).

vulgaris (Flem.). Garfish. Common off coast and in the Sound.

Scombresox (Lacép.).

saurus (Flem.). Skipper. One specimen found under the Hoe.

Exocœtus (Artedi).

volitans (Linn.). Greater flying fish. Recorded in Sutton Pool.

Engraulis (Cuv.).

encrasicholus (Cuv.). Anchovy. Taken off coast in drift nets occasionally.

Clupea (Artedi).

harengus (Linn.). Herring. Regular visitor.

pilchardus (Walb.). Pilchard. Regular visitor.

sprattus (Linn.). Sprat. Very common.

alosa (Linn.). Alewife. Common.

finta (Cuv.). Twaite shad. Occasional in the estuaries.

Anguilla (Belon).

vulgaris (Turt.). Eel. In estuaries, common.

Conger (Cuv.).

vulgaris (Cuv.). Conger. Common in deep water, on rocky ground.

\section{Lophobranchii.}

Syngnathus (Artedi).

acus (Linn.). Greater pipe fish. Common.

Nerophis (Rafin.).

lumbriciformis (Kröy). Worm-pipe fish. Common on shore.

\section{Plectognathi.}

Orthagoriscus (Bl. Schn.).

mola (Bl. Schn.). Short sun-fish. Sometimes seen off coast.

truncatus (Flem.). Oblong sun-fisia. Recorded off coast.

\section{List of Papers Consulted.}

No. 1. Alder and Hancock.- 'British Nudibranchiate Mollusca' (1845).

No. 2. Allman.- 'Monograph of Gymnoblastic Hydroids' (1872).

No. 3. Bate and Westwood.- 'British Sessile-eyed Crustacea' (1863-8).

No. 4. Bellamy.- 'The Natural History of South Devon' (1839).

No. 5. Bowerbank.- ' British Spongiadæ' (1864-82).

No. 6. 'British Association Reports.'- 'Reports on the Fauna of South Devon $(1867,1872)$.

No. 7. Couch.- 'British Fishes' (1877). 
No. 8. Darwin.- 'Monograph of the Cirripedia' (1881-3).

No. 9. Day.- 'British Fishes' (1880-4).

No. 10. Forbes. - 'British Starfishes,' 1841.

No. 11. Gosse.- 'British Sea Anemones and Corals' (1860).

No. 12. Hincks._- British Marine Polyzoa' (1880).

No. 13. Hunt.- “Notes on Tor Bay" ('Transactions of Devon Association,' 1878).

No. 14. Johnston. - 'Catalogue of British Non-Parasitical Worms in the Collection of the British Museum' (1865).

No. 15. Jeffreys.- 'British Conchology' (1862).

No. 16. McIntosh. - 'A Monograph of British Annelids' (1873-74).

No. 17. Parfitt.- 'Catalogue of the Fauna of Devon Zoophytes,' 1866.*

No. 18. Parfitt.- 'Catalogue of the Annelids of Devon,' 1867.

No. 19. Parfitt.- 'Catalogue of the Sponges of Devon,' 1868.

No. 20. Parfitt.--'Protozoa of Devonshire,' 1869.

No. 21. Parfitt.- 'Catalogue of the Crustacea-Podophthalmata of Devon,' 1870.

No. 22. Parfitt.- ' Fauna of Devon. Echinodermata,' 1872.

No. 23. Parfitt.- ' Fauna of Devon. Sessile-Eyed Crustacea, 1873.

No. 24. Parfitt.- ' Fauna of Devon. Conchologv,' 1874.

No. 25. Parfitt.- 'Fauna of Devon. Fishes,' 1875.

No. 26. Pennington.- 'British Zoophytes' (1885).

No. 27. Rogers.-List of Marine Animals for Sale.

No. 28. Yarrel.-'British Fishes' (1859).

No. 29. 'The Zoologist,' Oct., 1872 ; Jan., 1876; Sept., 1878; Aug., 1884.

* The remainder of Parfitt's papers all appear in the 'Transactions of the Devon Association.' The dates alone are mentioned. 